\title{
On possible time singular points and eventual regularity of weak solutions to the fractional Navier-Stokes equations
}

\author{
Quansen Jiu and Yanqing Wang \\ Communicated by Y. Charles Li, received July 3, 2014 \\ and, in revised form, August 21, 2014.
}

\begin{abstract}
In this paper, we intend to reveal how the fractional dissipation $(-\Delta)^{\alpha}$ affects the regularity of weak solutions to the $3 \mathrm{~d}$ generalized NavierStokes equations. Precisely, it will be shown that the $(5-4 \alpha) / 2 \alpha$ dimensional Hausdorff measure of possible time singular points of weak solutions on the interval $(0, \infty)$ is zero when $5 / 6 \leq \alpha<5 / 4$. To this end, the eventual regularity for the weak solutions is firstly established in the same range of $\alpha$. It is worth noting that when the dissipation index $\alpha$ varies from $5 / 6$ to $5 / 4$, the corresponding Hausdorff dimension is from 1 to 0 . Hence, it seems that the Hausdorff dimension obtained is optimal. Our results rely on the fact that the space $H^{\alpha}$ is the critical space or subcritical space to this system when $\alpha \geq 5 / 6$.
\end{abstract}

\section{Contents}

1. Introduction

2. Statement of the result

3. Well-posedness theory in $H^{\alpha}$ for $\alpha \geq 5 / 6$ and weak-strong uniqueness 327

4. Eventual regularity and Hausdorff dimension estimate 336

$\begin{array}{lll}\text { Appendix A. } & \text { Existence of weak solutions } & 339\end{array}$

References

\section{Introduction}

The incompressible time-dependent Navier-Stokes equations can be written as

$$
u_{t}-\nu \Delta u+u \cdot \nabla u+\nabla p=0, \operatorname{div} u=0,(x, t) \in \Omega \times(0, T),
$$

1991 Mathematics Subject Classification. Primary 35; Secondary 76.

Key words and phrases. Navier-Stokes equations, fractional dissipation, weak solutions, Hausdorff dimension, eventual regularity.

The first author was supported in part by National Natural Sciences Foundation of China (No. 11171229, No.11231006) and Project of Beijing Chang Cheng Xue Zhe. 
where the domain $\Omega$ denotes the whole space $\mathbb{R}^{n}$ or a torus $\mathbb{T}^{n}$ with $n \geq 2$. The unknown vector field $u$ describes velocity of the flow, the scalar function $p$ stands for the pressure of the fluid and the positive constant $\nu$ is the viscosity coefficient. We supplement problem (1.1) with the divergence-free initial data $u(x, 0)$.

It is well-known that the Navier-Stokes system enjoys two fundamental properties. One is energy equality (inequality)

$$
E(u)=\underset{t}{\operatorname{ess} \sup } \int_{\mathbb{R}^{n}}|u(x, t)|^{2} d x+2 \int_{0}^{\infty} \int_{\mathbb{R}^{n}}|\nabla u(x, \tau)|^{2} d x d \tau \leq \int_{\mathbb{R}^{n}}|u(x, 0)|^{2} d x,
$$

for smooth solution (weak solutions).

The other one is the scaling transformation, namely, if the couple $(u(x, t), p(x, t))$ solves problem $(1.1)$, then so does $\left(u_{\lambda}(x, t), p_{\lambda}(x, t)\right)$ with

$$
u_{\lambda}=\lambda u\left(\lambda x, \lambda^{2} t\right) \quad \text { and } \quad p_{\lambda}=\lambda^{2} p\left(\lambda x, \lambda^{2} t\right) .
$$

This induces us to investigate problem (1.1) in the critical spaces whose norm is invariant under scaling (1.3). Thus, a natural candidate is homogenous Sobolev space $\dot{H}^{(n-2) / 2}$ or Lebesgue space $L^{n}$. A Bananch space $X$ is said to be a supercritical space to (1.1) if $\left\|u_{\lambda}\right\|_{X} \rightarrow \infty$ as $\lambda$ tends to 0 . The rest are called as the subcritical spaces. We easily find that the energy space $L^{2}$ is a supercritical space to system (1.1) when the spatial dimension is strictly greater than two. Formally, based on (1.3), we see that

$$
\begin{aligned}
E\left(u_{\lambda}\right) & =\underset{\lambda^{2} t}{\operatorname{essup}} \int_{\mathbb{R}^{n}} \lambda^{2}\left|u\left(\lambda x, \lambda^{2} t\right)\right|^{2} d x+2 \int_{0}^{\infty} \int_{\mathbb{R}^{n}} \lambda^{4}\left|\nabla u\left(\lambda x, \lambda^{2} \tau\right)\right|^{2} d x d \tau \\
& =\lambda^{2-n} E(u),
\end{aligned}
$$

which implies that $E\left(u_{\lambda}\right) \rightarrow \infty$ as $\lambda \rightarrow 0$. In this sense, we may say that the $2 \mathrm{~d}$ Navier-Stokes equations is critical and the Navier-Stokes equations is supercritical when the spatial dimension is greater than or equal to three.

The global existence of weak solutions was successfully proved by Leray on the Cauchy problem and by Hopf for the Dirichlet problem. Both of them made full use of the energy inequality (1.2). Leray also proved that the weak solution of the 2d Navier-Stokes equations is regular in [11]. Although there are extensive studies on the regularity of weak solutions to the $3 \mathrm{~d}$ Navier-Stokes equations (See, e.g., $[\mathbf{2}, \mathbf{7}, \mathbf{8}, \mathbf{9}, \mathbf{1 7}, \mathbf{1 8}, \mathbf{1 9}, \mathbf{2 1}, \mathbf{2 2}, \mathbf{2 4}]$ ), the regularity of weak solutions is partially known until now. Particularly, Leray [11] proved that one dimension Lebesgue measure of the set of the possible time irregular points for weak solutions to the $3 \mathrm{~d}$ Navier-Stokes equations is zero. Moreover, Leray showed that every weak solution becomes smooth after a large time, namely, eventual regularity of the weak solutions. In [17], Scheffer improved Leray's upper bound of the Hausdorff dimension of the possible time singular set of weak solutions to $1 / 2$ (see also [8, Section 6] and [21, Chapter 5]). In this direction, Scheffer [18] also investigated the Hausdorff dimension of the space time singular set of weak solutions satisfying the local energy inequality, which is the so-called partial regularity of suitable weak solutions. The well-known Caffarelli-Kohn-Nirenberg theorem $[\mathbf{2}]$ showed that one dimensional Hausdorff measure of the possible space-time singular points of suitable weak solutions to the 3d Navier-Stokes equations is zero. For more general results on partial regularity of suitable weak solutions to the non-stationary and stationary 
Navier-Stokes system, we refer the reader to the paper [24] and the list of quotations there.

Generally speaking, it seems that there has not existed effective approach to deal with the supercritical equations so far. Concomitantly, an interesting question is: Could one modify the $3 \mathrm{~d}$ supercritical Navier-Stokes equations to become a critical (more regular)? As a matter of fact, this had been done by Lions in 1960s. More precisely, Lions $[\mathbf{1 2}, \mathbf{1 3}]$ introduced the following equations involving fractional dissipation

$$
\left\{\begin{array}{l}
u_{t}+\nu \Lambda^{2 \alpha} u+u \cdot \nabla u+\nabla p=0, \operatorname{div} u=0, \\
u(0)=u(0, x)
\end{array}\right.
$$

The operator $\Lambda^{2 \alpha}=(-\Delta)^{\alpha}$ is defined by $\widehat{\Lambda^{2 \alpha}} f(\xi)=|\xi|^{2 \alpha} \hat{f}(\xi)$ in the whole space, where $\hat{f}(\xi)=\frac{1}{(2 \pi)^{n}} \int_{\mathbb{R}^{n}} f(x) e^{-i \xi \cdot x} d x$; and by $\widehat{\Lambda^{2 \alpha} f}(k)=|\xi|^{2 \alpha} \hat{f}(k), k \in \mathbb{Z}^{n}$ on the torus, where $\hat{f}(k)=\frac{1}{(2 \pi)^{n}} \int_{\mathbb{T}^{n}} f(x) e^{-i k \cdot x} d x$. Here, $\alpha \geq 0$ is said to be the dissipation index. Exactly as the Navier-Stokes equations, note that if $u(x, t)$ solves (1.5), $u_{\lambda}=\lambda^{2 \alpha-1} u\left(\lambda x, \lambda^{2 \alpha} t\right)$ is also a solution of (1.5) for any $\lambda \in \mathbb{R}^{+}$. Formally, the corresponding energy is

$$
\begin{aligned}
E_{\alpha}\left(u_{\lambda}\right) & =\underset{\lambda^{2 \alpha} t}{\operatorname{ess} \sup } \int_{\mathbb{R}^{n}} \lambda^{4 \alpha-2}\left|u\left(\lambda x, \lambda^{2 \alpha} t\right)\right|^{2} d x+2 \int_{0}^{\infty} \int_{\mathbb{R}^{n}} \lambda^{6 \alpha-2}\left|\Lambda^{\alpha} u\left(\lambda x, \lambda^{2 \alpha} \tau\right)\right|^{2} d x d \tau \\
& =\lambda^{4 \alpha-(n+2)}\left\{\underset{t}{\operatorname{ess} \sup } \int_{\mathbb{R}^{n}}|u(x, t)|^{2} d x+2 \int_{0}^{\infty} \int_{\mathbb{R}^{n}}\left|\Lambda^{\alpha} u(x, \tau)\right|^{2} d x d \tau\right\} \\
& =\lambda^{4 \alpha-(n+2)} E_{\alpha}(u) .
\end{aligned}
$$

It follows that $E_{\alpha}\left(u_{\lambda}\right) \rightarrow \infty$ as $\lambda \rightarrow 0$ when $\alpha<(n+2) / 4$. Just as the above, we say that equations (1.5) is supercritical if $\alpha<(n+2) / 4$, critical for $\alpha=(n+2) / 4$ and subcritical with $\alpha>(n+2) / 4$. Lions obtained the global regular solution to the critical or subcritical equations (1.5) in [13].

When $1<\alpha<5 / 4$, the three-dimensioanl fractional Navier-Stokes system is still supercritical equations and their global well-posedness theories remain open. As pointed out by Katz and Pavlović in [10], any improvement in the exponent 5/4 could be viewed as genuine progress. It is remarkable that the Navier-Stokes equations with fractional dissipation have been studied from a mathematical viewpoint and some interesting results have been obtained in $[\mathbf{3}, \mathbf{1 0}, \mathbf{1 6}, \mathbf{2 3}, \mathbf{2 5}, \mathbf{2 6}, \mathbf{2 7}, \mathbf{2 8}$ ] and the references therein. The existence and uniqueness of solutions to the generalized Navier-Stokes equations (1.5) in Besov spaces were established by $\mathrm{Wu}$ in $[\mathbf{2 6}, \mathbf{2 7}]$. Recently, Tao $[\mathbf{2 3}]$ showed the global regularity for a logarithmically supercritical hyperdissipative Navier-Stokes system (see also [28]). In [10], it was shown that the Hausdorff dimension of the space singular set at time of first blow up for smooth solution to equations (1.5) is at most $5-4 \alpha$ as $1<\alpha<5 / 4$.

In this paper, one target of our work is to address the question: what is the precise effect of the fractional dissipation to the upper bound on the Hausdorff dimension of the potential time singular set for the weak solutions to (1.5). We shall prove that the $(5-4 \alpha) / 2 \alpha$ dimensional Hausdorff measure of possible time singular points of weak solutions on the interval $(0, \infty)$ is zero in the case when $5 / 6 \leq \alpha<5 / 4$. This result not only is an interpolation between the Scheffer's (Leray's) Hausdorff dimension of the possible time singular set of weak solutions 
to the Navier-Stokes equations $(\alpha=1)$ and Lions's global solvability for the hyperdissipative case $(\alpha \geq 5 / 4)$, but also further generalizes Scheffer's (Leray's) classic work in the sense that $5 / 6 \leq \alpha<1$. This gives an accurate relationship between the Hausdorff dimension of the potential time singular set of weak solutions and the dissipation index $\alpha$, which provides a perspective of the regularity of weak solutions to the fractional Navier-Stokes equations.

The other goal is to study the eventual regularity of weak solutions to (1.5). More precisely, with Leray's eventual regularization of weak solutions to the $3 \mathrm{~d}$ Navier-Stokes equations in mind, we want to explore how far we may go beyond the standard Laplacian dissipation $-\Delta$ and still prove the eventual regularity of weak solutions to the fractionally-dissipative Navier-Stokes equations. In particular, we can show the eventual regularity of weak solutions to (1.5) for $5 / 6 \leq \alpha$. It is natural to ask whether this result is true for $\alpha<5 / 6$. This is also partially motivated by the recently related work for the supercritical quasi-geostrophic type equation by M. Dabkowski [5], Miao and Xue [15] and L. Silvestre [20].

In what follows, we study (1.5) in a periodic domain $\mathbb{T}^{3}=\mathbb{R}^{3} / \mathbb{Z}^{3}$. The reason why we consider the periodic case is that it is not obvious whether the strong energy inequality of weak solutions to (1.5) is valid in whole space when $\alpha \neq 1$. Utilizing integration by parts, the spatial periodicity of the solution and the divergence free condition, we can derive that the solution $u$ of $(1.5)$ satisfies $\frac{d}{d t} \int_{\mathbb{T}^{3}} u(x, t) d x=0$. In addition, because of Galilean invariance of system (1.5), without loss of generality, we consider initial data of zero average, namely, $\int_{\mathbb{T}^{3}} u(x, 0) d x=0$. This yields that $\int_{\mathbb{T}^{3}} u(x, t) d x=0$ for any $t>0$. A function with vanishing spatial average on torus guarantees us to use its Sobolev (Gagliardo-Nirenberg) inequality like the whole space case. The reader is referred to $[\mathbf{1}]$ for more details.

This paper is organized as follows. In the second section, we shall present the definition of weak solutions to (1.5) for the initial data with finite-kinetic energy and state our main results. Section 3 is devoted to establishing the well-posedness theory with initial data in $H^{\alpha}$ for $\alpha \geq 5 / 6$. Then, the weak-strong uniqueness in the class $u \in L^{\infty}\left((0, T) ; H^{\alpha}\right) \cap L^{2}\left((0, T) ; H^{2 \alpha}\right)$ is discussed. Based on this, we could obtain the eventual regularity and the Hausdorff dimension of the possible singular time points of weak solutions in Section 4. Finally, for completeness, an appendix is dedicated to proving the existence of weak solutions to (1.5) for finite energy initial data.

Notation: The classical Sobolev norm $\|\cdot\|_{H^{s}}$ is defined as $\|f\|_{H^{s}}=\sum_{k \in \mathbb{Z}^{3}}(1+$ $\left.|k|^{2}\right)^{s}|\hat{f}(k)|^{2}, s \in \mathbb{R}$. We denote by $\dot{H}^{s}$ homogenous Sobolev spaces with the norm $\|f\|_{\dot{H}^{s}}=\sum_{k \in \mathbb{Z}^{3}}|k|^{2 s}|\hat{f}(k)|^{2}$. We shall denote by $\langle f, g\rangle$ the $L^{2}$ inner product of $f$ and $g$. For $p \in[1, \infty]$, the notation $L^{p}((0, T) ; X)$ stands for the set of measurable function on the interval $(0, T)$ with values in $X$ and $\|f\|_{X}$ belongs to $L^{p}(0, T)$. We will use $C$ to denote an absolute constant which may be different from line to line unless otherwise stated.

\section{Statement of the result}

In this section, we begin with the definition of weak solutions to the generalized Navier-Stokes equations (1.5). 
Definition 2.1. Let $u(0) \in L^{2}$ be a divergence-free vector. For any $T>0$, we say that $u$ is a weak solution to equations (1.5) with $\alpha>0$, if

(i) $u \in L^{\infty}\left((0, T) ; L^{2}(\Omega)\right) \cap L^{2}\left((0, T) ; H^{\alpha}(\Omega)\right)$;

(ii) $u$ solves (1.5) in the sense of distributions

$$
\int_{0}^{T}\left\langle u(t), \phi_{t}(t)\right\rangle-\nu\left\langle\Lambda^{\alpha} u(t), \Lambda^{\alpha} \phi(t)\right\rangle-\langle u(t) \cdot \nabla u(t), \phi(t)\rangle d t=-\langle u(0), \phi(0)\rangle,
$$

for all $\phi \in C_{0}^{\infty}((0, T) \times \Omega)$ with $\operatorname{div} \phi=0$.

(iii) $u$ fulfills the strong energy inequality

$$
\|u(t)\|_{L^{2}}^{2}+2 \nu \int_{\tau}^{t}\left\|\Lambda^{\alpha} u(s)\right\|_{L^{2}}^{2} d s \leq\|u(\tau)\|_{L^{2}}^{2},
$$

for $\tau=0$ or almost every $\tau>0$ and $t \in[\tau, T)$.

The global existence of weak solutions for the fractional Navier-Stokes equations (1.5) could be proved by the classical Faedo-Galerkin argument. For reader's convenience, we will sketch its proof in Appendix A.

The strong energy inequality plays an important role in the proof of eventual regularity and Hausdorff dimension estimate of possible time singular points. As said before, it is not clear whether the strong energy inequality of $(1.5)(\alpha \neq 1)$ is valid in the whole space since that when we apply compactness theorem to the approximation solution $u^{N}(x, t)$, we just obtain a subsequence of $u^{N}(x, t)$ which strongly converge to $u$ in $L_{l o c}^{2}\left(\mathbb{R}^{3}\right)$ for almost every $t \in[0, T]$ as $N$ tends to infinity. We refer the reader to Appendix A for the details. We would like to point out that the usual localization argument for the proof of the strong energy inequality to the 3d Navier-Stokes equations in the whole space or the exterior domain breaks down in the case that $\alpha \neq 1$ in (1.5) due to the presence of the nonlocal derivative.

Now we recall the definition of time singular point and Hausdorff dimension.

Definition 2.2 (Irregular Point, [11]). A time point $\tau<\infty$ is said to be an irregular point of the solution to the equations (1.5) if $u(s)$ is a regular solution on $(t, \tau) \times \Omega$, for some $t<\tau$, and it is impossible to extend $u(s)$ to a regular solution on $\left(t, \tau^{\prime}\right)$ with $\tau^{\prime}>\tau$.

Definition 2.3 (Hausdorff measure and Hausdorff dimension, [29]). For each $\gamma>0, \varepsilon>0$, and $E \subset \mathbb{R}^{n}$. Set

$\mathcal{H}^{\gamma}(E)$

$=\liminf _{\varepsilon \rightarrow 0}\left\{\sum_{i=1}^{\infty} \alpha(\gamma) 2^{-\gamma} \operatorname{diam}\left(B_{i}\right)^{\gamma}: E \subset \bigcup_{i=1}^{\infty} B_{i}, B_{i}\right.$ is an open ball and $\left.\operatorname{diam} B_{i}<\varepsilon\right\}$.

$\mathcal{H}^{\gamma}(E)$ is called the Hausdorff measure of $E$, where $\alpha(\gamma)$ denotes the volume of the unit ball in $\mathbb{R}^{n}$. For every set $E$, there is a non-negative number, $d=d(E)$, such that

$$
\begin{array}{ll}
\mathcal{H}^{\gamma}(E)=0 & \text { if } \gamma>d, \\
\mathcal{H}^{\gamma}(E)=\infty & \text { if } \gamma<d .
\end{array}
$$

the number $d(E)$ is called the Hausdorff dimension of $E$.

Let $\mathcal{I} \mathcal{R}$ stands for the possible time irregular points of weak solutions to (1.5) and the rest is denoted by $\mathcal{R}$. Our result is concerned with the estimate for the Hausdorff dimension of the set $\mathcal{I} \mathcal{R}$. 
TheOrem 2.4. For $5 / 6 \leq \alpha<5 / 4$, the $(5-4 \alpha) / 2 \alpha$ dimensional Hausdorff measure of the possible time singular points of weak solutions of (1.5) on the interval $(0, \infty)$ is zero, namely,

$$
\mathcal{H}^{\frac{5-4 \alpha}{2 \alpha}}(\mathcal{I} \mathcal{R})=0
$$

REMARK 2.5. In contrast to Katz and Pavlović's result that the Hausdorff dimension at the first time breakdown for the smooth solution to (1.5) is at most $5-4 \alpha$ for $1<\alpha<5 / 4$ in [10], our result is concerned with the Hausdorff dimension estimate of the potential time singular set for the finite energy weak solutions to (1.5) and is meaningful in the case $5 / 6 \leq \alpha<1$. It should be pointed out that the Hausdorff dimension of the potential time-space singular points for the (suitable) weak solutions to (1.5) has not been solved except $\alpha=1$.

Before we show the above theorem, actually, we first prove that

TheOREM 2.6. For $5 / 6 \leq \alpha<5 / 4$, there exists a constant $T^{*}>0$ such that the weak solutions to $(1.5)$ become strong solution on $\left(T^{*}, \infty\right)$.

REMARK 2.7. It is worth pointing out that our results are still valid under the assumption that the weak solutions satisfy the strong energy inequality in the whole space.

REMARK 2.8. According to (1.6), we notice that the $2 \mathrm{~d}$ fractional Navier-Stokes equations (1.5) is also a supercritical system in the case that $\alpha<1$. In the spirit of this paper, one could prove that the upper bound on the Hausdorff dimension of the potential time singular points of weak solutions to (1.5) for $2 / 3 \leq \alpha<1$ is $(2-2 \alpha) / \alpha$ and the eventual regularization of weak solutions is true when the spatial dimension is two. We leave this to the interested reader. More generally, the upper bound on the Hausdorff dimension of the possible time singular points of weak solutions to the $n$ dimensional generalized Navier-Stokes equations is $(n+2-4 \alpha) / 2 \alpha$ in the case that $(n+2) / 6 \leq \alpha<(n+2) / 4$.

To prove our results, we will employ some fundamental strategy in $[\mathbf{8}, \mathbf{9}, \mathbf{1 1}$, 17]. In Leray's pioneering work $[\mathbf{1 1}]$, he proved the global existence and uniqueness of solution to the $3 \mathrm{~d}$ Navier-Stokes equations with initial velocity $u(0) \in H^{1}$ provided $\|u(0)\|_{L^{2}}^{1 / 2}\|\nabla u(0)\|_{L^{2}}^{1 / 2}$ being sufficiently small and the local solvability when the initial data belongs to space $H^{1}$ without smallness assumption. See $[\mathbf{8}, \mathbf{9}]$ for a more modern exposition. Leray's eventual regularization of weak solutions relied on the $H^{1}$ well-posedness result. In addition, it is worth mentioning that

$$
\|u(0)\|_{\dot{H}^{1 / 2}} \leq C\|u(0)\|_{L^{2}}^{\frac{1}{2}}\|\nabla u(0)\|_{L^{2}}^{\frac{1}{2}}
$$

is invariant under the scaling (1.4).

A key observation is an analogous inequality of (2.2)

$$
\|u\|_{\dot{H} \frac{5-4 \alpha}{2}} \leq C\|u\|_{L^{2}}^{\frac{6 \alpha-5}{2 \alpha}}\left\|\Lambda^{\alpha} u\right\|_{L^{2}}^{\frac{5-4 \alpha}{2 \alpha}}, 5 / 6 \leq \alpha \leq 5 / 4 .
$$

Notice that $\dot{H}^{\frac{5-4 \alpha}{2}}$ appearing in the last inequality is the critical space to (1.5) due to the fact that $\left\|u_{\lambda}\right\|_{\dot{H}^{\frac{5-4 \alpha}{2}}\left(\mathbb{R}^{3}\right)}=\|u\|_{\dot{H}^{\frac{5-4 \alpha}{2}}\left(\mathbb{R}^{3}\right)}$ where $u_{\lambda}=\lambda^{2 \alpha-1} u(\lambda x)$, from which we see that the energy space $L^{2}$ is the critical space to (1.5) when $\alpha=5 / 4$. Another interesting ingredient of the latter inequality is the borderline case that $\alpha=5 / 6$, which suggests us that $5 / 6$ is a probable endpoint of our discussion. Indeed, we would like to point out that $H^{\alpha}$ is the subcritical space to (1.5) when 
$\alpha>5 / 6$ in the sense that $\left\|u_{\lambda}\right\|_{\dot{H}^{\alpha}\left(\mathbb{R}^{3}\right)}$ converges to 0 as $\lambda$ tends to 0 . Thence, we obtain a strong solution when we solves (1.5) with $\alpha \geq 5 / 6$ in $H^{\alpha}$.

More precisely, we shall establish the local existence for arbitrarily large data in space $H^{\alpha}$ and the global well-posedness of (1.5) under the assumption that the quantity $\|u(0)\|_{L^{2}}^{(6 \alpha-5) / 2 \alpha}\left\|\Lambda^{\alpha} u(0)\right\|_{2}^{(5-4 \alpha) / 2 \alpha}$ is small enough. To this end, we exploit some new estimates to (1.5) compared to the previous estimates presented in $[\mathbf{1 3}, \mathbf{2 7}]$. Next, we show that this strong solution coincides with the weak solutions associated with the same initial data. Roughly speaking, small solution in $H^{\alpha}$, weak-strong uniqueness, and the strong energy inequality (2.1) mean the eventual regularization of weak solutions. Thus, we complete the proof of theorem 2.6.

As a critical by-product of well-posedness result in $H^{\alpha}$, we could deduce that the blow-up rate

$$
\left\|\Lambda^{\alpha} u(t)\right\|_{L^{2}} \geq \frac{C \nu^{\frac{5-2 \alpha}{4 \alpha}}}{\left(t_{0}-t\right)^{\frac{6 \alpha-5}{4 \alpha}}}, t<t_{0}, 5 / 6<\alpha<5 / 4,
$$

for $t_{0}$ to be a potential irregular point. This enables us to achieve the Hausdorff dimensional estimate of possible time singular points of weak solutions on the interval $(0, \infty)$.

\section{Well-posedness theory in $H^{\alpha}$ for $\alpha \geq 5 / 6$ and weak-strong uniqueness}

3.1. Strong solution. The following is about the existence and uniqueness of the solution to (1.5) for $\alpha \geq 5 / 6$ with the initial data in $H^{\alpha}$. Notice that global well-posedness of (1.5) for small initial data belonging to $\dot{H}^{5 / 6}$ has been established in the sense that $u \in L^{\infty}\left(0, \infty ; \dot{H}^{5 / 6}\right) \cap L^{2}\left(0, \infty ; \dot{H}^{5 / 3}\right)$ by $\mathrm{Wu}$ in [27, Theorem 6.1$]$, where we have used the fact that homogeneous space $\dot{H}^{5 / 6}$ and homogeneous Besov space $\dot{B}_{2,2}^{5 / 6}$ coincide. For the local well-posedness for large initial data in $\dot{H}^{5 / 6}$, see also [27, Theorem 6.2]. The solution constructed by Wu is also a weak solution when the initial data belongs to $H^{5 / 6}$. This fact is clear since the domain considered here is periodic. Consequently, we mainly pay our attention on the case when $\alpha>5 / 6$ below.

Proposition 3.1 (Strong solution). For every $u(0) \in H^{\alpha}(5 / 6<\alpha \leq 5 / 4)$ with divergence-free, there exists a constant

$$
T<\frac{\nu^{\frac{5-2 \alpha}{6 \alpha-5}}}{\frac{2 \alpha C}{6 \alpha-5}\left\|\Lambda^{\alpha} u(0)\right\|_{L^{2}}^{\frac{4 \alpha}{6 \alpha-5}}}
$$

such that the system (1.5) admits a unique solution $u$ in

$$
L^{\infty}\left((0, T) ; H^{\alpha}\right) \cap L^{2}\left((0, T) ; H^{2 \alpha}\right) .
$$

Moreover, let $5 / 6<\alpha<5 / 4$ and assume that the initial data satisfies

$$
\|u(0)\|_{L^{2}}^{\frac{6 \alpha-5}{2 \alpha}}\left\|\Lambda^{\alpha} u(0)\right\|_{L^{2}}^{\frac{5-4 \alpha}{2 \alpha}}<C_{1}^{-1} \nu
$$

for some constant $C_{1}$ depending on the domain $\Omega$. Then $T$ can be chosen as arbitrary positive constants.

REMARK 3.2. After we finished this paper, we notice that the well-posedness theory in $\dot{H}^{\frac{5-4 \alpha}{2}}$ to equations (1.5) with $\alpha \geq 5 / 6$ had been established in [27]. Compared with the method adopted in [27], our method is elementary and avoids 
the highly-sophisticated tools from harmonic analysis. More importantly, as a byproduct of our analysis, we could deduce a necessary condition for some time $t$ to be a potential blow up time which plays an important role in the proof of Theorem 2.4. For the detail, see Proposition 4.2.

Proof. The existence proof is based on the approximate system below

$$
\partial_{t} u^{N}+\nu \Lambda^{2 \alpha} u^{N}+P_{N}\left(u^{N} \cdot \nabla u^{N}\right)+P_{N} \nabla p^{N}=0, \operatorname{div} u^{N}=0
$$

together with the initial condition

$$
u^{N}(x, 0)=P_{N} u(x, 0),
$$

where $P_{N} f(x)=\mathcal{F}^{-1}\left(1_{k \leq N}(k) \hat{u}(k)\right)$. For its detail, see the Appendix A.

Step 1: Local well-posedness

Taking the inner product of approximate equations with $u^{N}$ and integrating by parts, we know that

$$
\frac{1}{2} \frac{d}{d t}\left\|u^{N}(t)\right\|_{L^{2}}^{2}+\nu\left\|\Lambda^{\alpha} u^{N}(t)\right\|_{L^{2}}^{2}=0
$$

which implies that

$$
\frac{1}{2}\left\|u^{N}(t)\right\|_{L^{2}}^{2}+\nu \int_{0}^{t}\left\|\Lambda^{\alpha} u^{N}(s)\right\|_{L^{2}}^{2} d s=\frac{1}{2}\left\|u^{N}(0)\right\|_{L^{2}}^{2} \leq \frac{1}{2}\|u(0)\|_{L^{2}}^{2} .
$$

Similarly, multiplying (1.5) by $\Lambda^{2 \alpha} u$, we arrive at

$$
\frac{1}{2} \frac{d}{d t}\left\|\Lambda^{\alpha} u^{N}(t)\right\|_{L^{2}}^{2}+\nu\left\|\Lambda^{2 \alpha} u^{N}(t)\right\|_{L^{2}}^{2}=\left\langle u^{N}(t) \cdot \nabla u^{N}(t), \Lambda^{2 \alpha} u^{N}(t)\right\rangle .
$$

By virtue of the Gagliardo-Nirenberg inequality,

$$
\left\|u^{N}(t)\right\|_{L^{\frac{6}{3-2 \alpha}}} \leq C\left\|\Lambda^{\alpha} u^{N}(t)\right\|_{L^{2}},
$$

and

$$
\left\|\nabla u^{N}(t)\right\|_{L^{\frac{3}{\alpha}}} \leq C\left\|\Lambda^{\alpha} u^{N}(t)\right\|_{L^{2}}^{\frac{6 \alpha-5}{2 \alpha}}\left\|\Lambda^{2 \alpha} u^{N}(t)\right\|_{L^{2}}^{\frac{5-4 \alpha}{2 \alpha}} .
$$

we obtain

$$
\begin{aligned}
\left|\left\langle u^{N}(t) \cdot \nabla u^{N}(t), \Lambda^{2 \alpha} u^{N}(t)\right\rangle\right| & \leq\left\|u^{N}(t)\right\|_{L^{\frac{6}{3-2 \alpha}}}\left\|\nabla u^{N}(t)\right\|_{L^{\frac{3}{\alpha}}(\Omega)}\left\|\Lambda^{2 \alpha} u^{N}(t)\right\|_{L^{2}} \\
& \leq C\left\|\Lambda^{\alpha} u^{N}(t)\right\|_{L^{2}}\left\|\Lambda^{\alpha} u^{N}(t)\right\|_{L^{\frac{2 \alpha}{2 \alpha}}}^{\frac{6 \alpha-5}{2 \alpha}} u^{N}(t)\left\|_{L^{\frac{5-2 \alpha}{2 \alpha}}}^{2 \frac{2 \alpha-5}{2}}\right\| \Lambda^{\alpha} u^{N}(t) \|_{L^{2}}^{\frac{2(8 \alpha-5}{6 \alpha-5}} \\
& \leq \frac{1}{2} \nu\left\|\Lambda^{2 \alpha} u^{N}(t)\right\|_{L^{2}}^{2}+C \nu^{\frac{2 \alpha-5}{6 \alpha-5}}
\end{aligned}
$$

where we used Hölder's inequality and Young's inequality.

Plugging this into bound (3.3) gives

$$
\frac{d}{d t}\left\|\Lambda^{\alpha} u^{N}(t)\right\|_{L^{2}}^{2}+\nu\left\|\Lambda^{2 \alpha} u^{N}(t)\right\|_{L^{2}}^{2} \leq C \nu^{\frac{2 \alpha-5}{6 \alpha-5}}\left\|\Lambda^{\alpha} u^{N}(t)\right\|_{L^{2}}^{\frac{2(8 \alpha-5)}{6 \alpha-5}}
$$


which implies that

$$
\begin{aligned}
\left\|\Lambda^{\alpha} u^{N}(t)\right\|_{L^{2}} & \leq \frac{\left\|\Lambda^{\alpha} u^{N}(0)\right\|_{L^{2}}}{\left(1-\frac{2 \alpha C}{6 \alpha-5} \nu^{\frac{2 \alpha-5}{6 \alpha-5}}\left\|\Lambda^{\alpha} u^{N}(0)\right\|_{L^{2}}^{\frac{4 \alpha}{6 \alpha-5}} t\right)^{\frac{6 \alpha-5}{4 \alpha}}} \\
& \leq \frac{\left\|\Lambda^{\alpha} u(0)\right\|_{L^{2}}}{\left(1-\frac{2 \alpha C}{6 \alpha-5} \nu^{\frac{2 \alpha-5}{6 \alpha-5}}\left\|\Lambda^{\alpha} u(0)\right\|_{L^{2}}^{\frac{4 \alpha}{6 \alpha-5}} t\right)^{\frac{6 \alpha-5}{4 \alpha}}} \\
& \leq\left\|\Lambda^{\alpha} u(0)\right\|_{L^{2}}+\frac{C\left\|\Lambda^{\alpha} u(0)\right\|_{L^{2}}^{2} \nu^{\frac{2 \alpha-5}{4 \alpha}} t^{\frac{6 \alpha-5}{4 \alpha}}}{\left(1-\frac{2 \alpha C}{6 \alpha-5} \nu^{\frac{2 \alpha-5}{6 \alpha-5}}\left\|\Lambda^{\alpha} u(0)\right\|_{L^{2}}^{\frac{4 \alpha}{6 \alpha-5}} t\right)^{\frac{6 \alpha-5}{4 \alpha}}}
\end{aligned}
$$

where we have used the fact that $\left\|u^{N}(0)\right\|_{L^{2}} \leq\|u(0)\|_{L^{2}}$ and the elementary inequality $(a-b)^{\beta} \geq a^{\beta}-b^{\beta}$ for $a>b>0$ and $0<\beta<1$.

Thus, there exists a constant $T:=T_{*}$ with

$$
T_{*}=\frac{\nu^{\frac{5-2 \alpha}{6 \alpha-5}}}{\frac{2 \alpha C}{6 \alpha-5}\left\|\Lambda^{\alpha} u(0)\right\|_{L^{2}}^{\frac{4 \alpha}{6 \alpha-5}}} .
$$

such that

$$
\left\|\Lambda^{\alpha} u^{N}(t)\right\|_{L^{2}}^{2} \leq C, \quad t \in\left[0, T_{*}\right),
$$

where the constant $C$ is independent of $N$. Therefore, we deduce the uniformly bounded estimate that

$$
\left\|\Lambda^{\alpha} u^{N}(t)\right\|_{L^{2}}^{2}+\nu \int_{0}^{t}\left\|\Lambda^{2 \alpha} u^{N}(s)\right\|_{L^{2}}^{2} d s \leq C, t<T^{*} .
$$

Furthermore, by $(3.2)$, for any $g \in L^{2}\left([0, t] ; L^{2}\right)$, note that

$$
\begin{aligned}
\int_{0}^{t}\left\langle\Lambda^{2 \alpha} u^{N}(s), g(s)\right\rangle d s & \leq \int_{0}^{t}\left\|\Lambda^{\alpha} u^{N}(s)\right\|_{L^{2}}\left\|\Lambda^{\alpha} g(s)\right\|_{L^{2}} d s \\
& \leq\left(\int_{0}^{t}\left\|\Lambda^{\alpha} u^{N}(s)\right\|_{L^{2}}^{2} d s\right)^{1 / 2}\left(\int_{0}^{t}\left\|\Lambda^{\alpha} g(s)\right\|_{L^{2}}^{2} d s\right)^{1 / 2} \\
& \leq C\|u(0)\|_{L^{2}}\|g(s)\|_{L^{2}\left([0, t] ; L^{2}\right)}
\end{aligned}
$$

which yields that $\Lambda^{2 \alpha} u^{N} \in L^{2}\left([0, t] ; L^{2}\right)$.

By the Gagliardo-Nirenberg inequality, we have

$$
\left\|\nabla u^{N}\right\|_{L^{\frac{3}{\alpha}}} \leq C\left\|\Lambda^{\alpha} u^{N}\right\|_{L^{2}}^{\frac{6 \alpha-5}{2 \alpha}}\left\|\Lambda^{2 \alpha} u^{N}\right\|_{L^{2}}^{\frac{5-4 \alpha}{2 \alpha}},
$$

and

$$
\left\|u^{N}\right\|_{L^{\frac{6}{3-2 \alpha}}} \leq C\left\|\Lambda^{\alpha} u^{N}\right\|_{L^{2}}
$$


and (3.2), we infer that

$$
\begin{aligned}
& \int_{0}^{t}\left\langle P_{N}\left(u^{N} \cdot \nabla u^{N}\right), g\right\rangle d s \\
\leq & \int_{0}^{t}\left\|u^{N}\right\|_{L^{\frac{6}{3-2 \alpha}}}\left\|\nabla u^{N}\right\|_{L^{\frac{3}{\alpha}}}\|g\|_{L^{2}} d s \\
\leq & \int_{0}^{t}\left\|\Lambda^{\alpha} u^{N}\right\|_{L^{2}}\left\|\Lambda^{\alpha} u^{N}\right\|_{L^{\frac{2 \alpha}{2 \alpha}}}^{\frac{6 \alpha-5}{2 \alpha}} \Lambda^{2 \alpha} u^{N}\left\|_{L^{\frac{5-4 \alpha}{2 \alpha}}}^{\frac{2 \alpha}{2}}\right\| g \|_{L^{2}} d s \\
\leq & C\left(\int_{0}^{t}\left\|\Lambda^{\alpha} u^{N}\right\|_{L^{2}}^{2}\left\|\Lambda^{\alpha} u^{N}\right\|_{L^{2}}^{\frac{6 \alpha-5}{\alpha}}\left\|\Lambda^{2 \alpha} u^{N}\right\|_{L^{2}}^{\frac{5-4 \alpha}{\alpha}} d s\right)^{\frac{1}{2}}\left(\int_{0}^{t}\|g\|_{L^{2}}^{2} d s\right)^{\frac{1}{2}} \\
\leq & C\|u(0)\|_{L^{2}}\left(\int_{0}^{t}\left\|\Lambda^{2 \alpha} u^{N}\right\|_{L^{2}}^{\frac{5-4 \alpha}{\alpha}} d s\right)^{\frac{1}{2}}\|g\|_{L^{2}\left([0, t] ; L^{2}\right)} \\
\leq & C\left(T_{*}\right)\|u(0)\|_{L^{2}}^{2}\|g\|_{L^{2}\left([0, t] ; L^{2}\right)},
\end{aligned}
$$

where we have used (3.2) and (3.5). This in turn implies that $P_{N}\left(u^{N} \cdot \nabla u^{N}\right) \in$ $L^{2}\left([0, t] ; L^{2}\right)$ since $5 / 6 \leq \alpha \leq 5 / 4$.

Using the pressure equation

$$
\Delta p^{N}=-\sum_{i, j} \partial_{i} \partial_{j}\left(u_{i}^{N} u_{j}^{N}\right)
$$

and the boundedness of Riesz transforms on $L^{q}$ for any $1<q<\infty$, we have $\left\|P_{N} \nabla p^{N}\right\|_{L^{2}} \leq\left\|u^{N} \cdot \nabla u^{N}\right\|_{L^{2}}$, from which, exactly as in the derivation of (3.6), yields that $P_{N} \nabla p^{N} \in L^{2}\left([0, t] ; L^{2}\right)$.

Collecting the uniform estimate and recalling that $\partial_{t} u^{N}=-\nu \Lambda^{2 \alpha} u^{N}-P_{N}(u$. $\left.\nabla u^{N}\right)-P_{N} \nabla p_{N}$, we obtain the desired estimate $\partial_{t} u^{N} \in L^{2}\left([0, t] ; L^{2}\right)$. This together with (3.5), by means of Aubin-Lions Lemma ([22, Theorem 2.1, p.184]), we could claim that $u_{N}$ strongly converge to $u$ as $N$ tends to infinity in $L^{2}\left((0, T) ; H^{\alpha-\epsilon}\right)$ for any $\epsilon>0$. By Fatou's Lemma, we get that $u \in L^{\infty}\left((0, T) ; H^{\alpha}\right) \cap L^{2}\left((0, T) ; H^{2 \alpha}\right)$. We finish the proof of local existence. The uniqueness is a consequence of the weakstrong uniqueness Proposition 3.4 whose proof is postponed to the next subsection 3.2 .

Step 2: Global well-posedness for small initial data.

It suffices to show the uniform boundedness of $\left\|\Lambda^{\alpha} u^{N}(t)\right\|_{L^{2}}^{2}$ where $t \in\left[0, T^{\prime}\right]$ for any given constant $T^{\prime}$. Indeed, having this uniform estimate in hand, by means of (3.4), one immediately obtains the uniform estimate of $\int_{0}^{t}\left\|\Lambda^{2 \alpha} u^{N}(s)\right\|_{L^{2}}^{2} d s$ for $t<T^{\prime}$. The rest part of passing to the limit of the approximations solution is analogous to the one of local well-posedness.

To this end, using once again the Gagliardo-Nirenberg inequality, we obtain

$$
\left\|u^{N}\right\|_{L^{\frac{3}{2 \alpha-1}}} \leq C\left\|u^{N}\right\|_{L^{2}}^{\frac{6 \alpha-5}{2 \alpha}}\left\|\Lambda^{\alpha} u^{N}\right\|_{L^{2}}^{\frac{5-4 \alpha}{2 \alpha}} \text {, and }\left\|\nabla u^{N}\right\|_{L^{\frac{6}{5-4 \alpha}}} \leq C\left\|\Lambda^{2 \alpha} u^{N}\right\|_{L^{2}},
$$

which yields

$$
\begin{aligned}
\left|\left\langle u^{N} \cdot \nabla u^{N}, \Lambda^{2 \alpha} u^{N}\right\rangle\right| & \leq\left\|u^{N}\right\|_{L^{\frac{3}{2 \alpha-1}}}\left\|\nabla u^{N}\right\|_{L^{\frac{6}{5-4 \alpha}}}\left\|\Lambda^{2 \alpha} u^{N}\right\|_{L^{2}} \\
& \leq C_{1}\left\|u^{N}\right\|_{L^{2}}^{\frac{6 \alpha-5}{2 \alpha}}\left\|\Lambda^{\alpha} u^{N}\right\|_{L^{2}}^{\frac{5-4 \alpha}{2 \alpha}}\left\|\Lambda^{2 \alpha} u^{N}\right\|_{L^{2}}^{2} .
\end{aligned}
$$

Substituting the latter inequality into (3.3), we get

$$
\text { 7) } \frac{1}{2} \frac{d}{d t}\left\|\Lambda^{\alpha} u^{N}(t)\right\|_{L^{2}}^{2}+\left(\nu-C_{1}\left\|u^{N}(t)\right\|_{L^{2}}^{\frac{6 \alpha-5}{2 \alpha}}\left\|\Lambda^{\alpha} u^{N}(t)\right\|_{L^{2}}^{\frac{5-4 \alpha}{2 \alpha}}\right)\left\|\Lambda^{2 \alpha} u^{N}(t)\right\|_{L^{2}}^{2} \leq 0 .
$$


Assume for a while we have proved that

$$
\left\|u^{N}(t)\right\|_{L^{2}}^{\frac{6 \alpha-5}{2 \alpha}}\left\|\Lambda^{\alpha} u^{N}(t)\right\|_{L^{2}}^{\frac{5-4 \alpha}{2 \alpha}}<C_{1}^{-1} \nu, \text { for each } t \in\left[0, T^{\prime}\right) .
$$

Therefore, it follows form (3.7) that $\frac{d}{d t}\left\|\Lambda^{\alpha} u^{N}(t)\right\|_{L^{2}}^{2} \leq 0\left(t \in\left[0, T^{\prime}\right)\right)$, which yields the desired estimate.

Now we need to prove the equality (3.8) we have assumed. By the hypothesis on initial condition (3.1), we could suppose that $T_{1}<T^{\prime}$ is the first time such that

$$
\left\|u^{N}\left(T_{1}\right)\right\|_{L^{2}}^{\frac{6 \alpha-5}{2 \alpha}}\left\|\Lambda^{\alpha} u^{N}\left(T_{1}\right)\right\|_{L^{2}}^{\frac{5-4 \alpha}{2 \alpha}}=C_{1}^{-1} \nu .
$$

Consequently, for any $s \in\left[0, T_{1}\right]$, we have $\left\|u^{N}(s)\right\|_{L^{2}}^{\frac{6 \alpha-5}{2 \alpha}}\left\|\Lambda^{\alpha} u^{N}(s)\right\|_{L^{2}}^{\frac{5-4 \alpha}{2 \alpha}} \leq C_{1}^{-1} \nu$. Thanks to (3.2) and (3.7), we obtain

$$
\left\|u^{N}\left(T_{1}\right)\right\|_{L^{2}} \leq\left\|u^{N}(0)\right\|_{L^{2}} \leq\|u(0)\|_{L^{2}}
$$

and

$$
\left\|\Lambda^{\alpha} u^{N}\left(T_{1}\right)\right\|_{L^{2}} \leq\left\|\Lambda^{\alpha} u^{N}(0)\right\|_{L^{2}} \leq\left\|\Lambda^{\alpha} u(0)\right\|_{L^{2}},
$$

since $\frac{d}{d s}\left\|\Lambda^{\alpha} u\left(T_{1}\right)\right\|_{L^{2}}^{2} \leq 0$.

However, through a simple calculation, we see that

$$
\nu-C_{1}\left\|u^{N}\left(T_{1}\right)\right\|_{L^{2}}^{\frac{6 \alpha-5}{2 \alpha}}\left\|\Lambda^{\alpha} u^{N}\left(T_{1}\right)\right\|_{L^{2}}^{\frac{5-4 \alpha}{2 \alpha}} \geq \nu-C_{1}\left\|u^{N}(0)\right\|_{L^{2}}^{\frac{6 \alpha-5}{2 \alpha}}\left\|\Lambda^{\alpha} u^{N}(0)\right\|_{L^{2}}^{\frac{5-4 \alpha}{2 \alpha}}>0 .
$$

which contradicts (3.9). Thus, the claim (3.8) is proved.

REMARK 3.3. A straightforward consequence of the above proof is that we provide an alternative approach to show Lions's global solvability to the case $\alpha=$ $5 / 4$. Indeed, it follows from the equation (3.4) and the Gronwall's inequality that

$$
\left\|\Lambda^{5 / 4} u^{N}(t)\right\|_{L^{2}}^{2} \leq\left\|\Lambda^{5 / 4} u^{N}(0)\right\|_{L^{2}}^{2} e^{C \int_{0}^{t}\left\|\Lambda^{5 / 4} u^{N}(s)\right\|_{L^{2}}^{2} d s} \leq\left\|\Lambda^{5 / 4} u(0)\right\|_{L^{2}}^{2} e^{C\|u(0)\|_{L^{2}}^{2}} .
$$

Thus, there exists a global strong solution to (1.5) without without any smallness restriction on the initial data belonging to space $H^{5 / 4}$ when $\alpha=5 / 4$. (It is well known that the finite weak solution $u \in L^{\infty}\left((0, T) ; L^{2}\right) \cap L^{2}\left((0, T) ; H^{5 / 4}\right)$ is a regular solution to the (1.5) for $\alpha=5 / 4$. Here, we show that the weak solution has higher regularity when the initial data is more regular in the case $\alpha=5 / 4$.)

Next we shall prove that the strong solution $u \in L^{\infty}\left((0, T) ; H^{\alpha}\right) \cap L^{2}\left((0, T) ; H^{2 \alpha}\right)$ constructed above and the case $\alpha=5 / 6$ in $[\mathbf{2 7}]$ coincides with the weak solution $v \in L^{\infty}\left((0, T) ; L^{2}\right) \cap L^{2}\left((0, T) ; H^{\alpha}\right)$ associated with the same initial data for $5 / 6 \leq \alpha<5 / 4$. Here, we basically follow the pathway of $[\mathbf{4}, \mathbf{6}, \mathbf{8}, \mathbf{1 4}, \mathbf{1 9}]$ to obtain the weak-strong uniqueness in the class $u \in L^{\infty}\left((0, T) ; H^{\alpha}\right) \cap L^{2}\left((0, T) ; H^{2 \alpha}\right)$.

\subsection{Weak-strong uniqueness.}

Proposition 3.4. Let $5 / 6 \leq \alpha \leq 5 / 4$. From Proposition 3.1 in the last subsection and Theorem 6.2 in [27], there exist a solution $u \in L^{\infty}\left((0, T) ; H^{\alpha}\right) \cap$ $L^{2}\left((0, T) ; H^{2 \alpha}\right)$ to $(1.5)$ for a constant $T>0$ associated with data $u(0)$ in $H^{\alpha}$. Let $v$ be any weak solution to (1.5) with initial condition $u(0)$. Then $u=v$ for a.e. $x \in \Omega, t>0$.

In order to show Proposition 3.4, we need the following lemmas. 
Lemma 3.5. Let $v$ be a weak solution to $(1.5)$ in $(0, T) \times \Omega$. Then $v$ can be redefined on a set of zero Lebesgue measure in such a way that $v(t) \in L^{2}$ for all $t \in(0, T)$ and satisfies the identity

$$
\int_{s}^{t}\left\langle v, \varphi_{\tau}\right\rangle-\nu\left\langle\Lambda^{\alpha} v, \Lambda^{\alpha} \varphi\right\rangle-\langle v \cdot \nabla v, \varphi\rangle d \tau=\langle v(t), \varphi(t)\rangle-\langle v(s), \varphi(s)\rangle,
$$

for all $s \in[0, t], t<T$ and all $\varphi \in C^{\infty}\left((0, T) \times C_{0}^{\infty}\right)$.

The proof of this lemma is similar to the classical Navier-Stokes equations $([\mathbf{8}, \mathbf{1 4}, \mathbf{1 9}])$ and we omit the details here. The following is about an approximation lemma.

Lemma 3.6 ([14], Lemma 2.1). Suppose that $X$ is a Banach space, $w \in$ $L^{q}((0, T) ; X), 1 \leq q<\infty, w_{\rho}(s)=\int_{0}^{t} J_{\rho}(s-\tau) w(\tau) d \tau$, where $J_{\rho}(t)=\rho^{-1} J(t / \rho)$ and $J$ is an even nonnegative smooth function with $\int_{-\infty}^{\infty} J(s) d s=1$ and $J \in$ $C_{0}^{\infty}(-1,1)$. Then $w_{\rho} \in C^{1}([0, t] ; X)$ and

$$
\lim _{\rho \rightarrow 0}\left\|w_{\rho}-w\right\|_{L^{q}((0, T) ; X)}=0 .
$$

The main proof of Proposition 3.4 relies on the following key lemma.

Lemma 3.7. Suppose that $u$ and $v$ are two weak solutions of (1.5) in Proposition 3.4 and let $w=u-v$. Then for any $T>0$, there holds

$\langle u(t), v(t)\rangle+2 \nu \int_{0}^{t}\left\langle\Lambda^{\alpha} u(s), \Lambda^{\alpha} v(s)\right\rangle d s=\langle u(0), v(0)\rangle-\int_{0}^{t}\langle w(s) \cdot \nabla u(s), w(s)\rangle d s$.

Proof. It follows from Lemma 3.6 that

$$
u_{\rho} \in H^{1}\left([0, T] ; H^{2 \alpha}\right), v_{\rho} \in H^{1}\left([0, T] ; H^{\alpha}\right)
$$

and

$$
\lim _{\rho \rightarrow 0}\left\|u_{\rho}-u\right\|_{L^{2}\left((0, T) ; H^{2 \alpha}\right)}=0, \lim _{\rho \rightarrow 0}\left\|v_{\rho}-v\right\|_{L^{2}\left((0, T) ; H^{\alpha}\right)}=0 .
$$

Due to the fact that $C_{0}^{\infty}(\Omega)$ is dense in $H^{\alpha}(\Omega)\left(H^{2 \alpha}(\Omega)\right)$ and Lemma 2.2 in [14], there exist $u_{\rho}^{k}, v_{\rho}^{k} \in C^{\infty}\left([0, t] ; C_{0}^{\infty}\right)$ such that

$$
\lim _{k \rightarrow \infty}\left\|u_{\rho}^{k}-u_{\rho}\right\|_{H^{1}\left([0, t] ; H^{2 \alpha}\right)}=0, \lim _{k \rightarrow \infty}\left\|v_{\rho}^{k}-v_{\rho}\right\|_{H^{1}\left([0, t] ; H^{\alpha}\right)}=0 .
$$

Choosing $u_{\rho}^{k}$ and $v_{\rho}^{k}$ as test functions in (3.10) respectively, one has

$$
\begin{aligned}
& \int_{0}^{t}\left\langle v, \partial_{s} u_{\rho}^{k}\right\rangle-\nu\left\langle\Lambda^{\alpha} v, \Lambda^{\alpha} u_{\rho}^{k}\right\rangle-\left\langle v \cdot \nabla v, u_{\rho}^{k}\right\rangle d s=\left\langle v(t), u_{\rho}^{k}(t)\right\rangle-\left\langle v(0), u_{\rho}^{k}(0)\right\rangle, \\
& \int_{0}^{t}\left\langle u, \partial_{s} v_{\rho}^{k}\right\rangle-\nu\left\langle\Lambda^{\alpha} u, \Lambda^{\alpha} v_{\rho}^{k}\right\rangle-\left\langle u \cdot \nabla u, v_{\rho}^{k}\right\rangle d s=\left\langle u(t), v_{\rho}^{k}(t)\right\rangle-\left\langle u(0), v_{\rho}^{k}(0)\right\rangle .
\end{aligned}
$$

Now we take the limit in the above equations. By (3.12), it is direct to get

$$
\lim _{k \rightarrow \infty} \int_{0}^{t}\left\langle v, \partial_{s} u_{\rho}^{k}\right\rangle d s=\int_{0}^{t}\left\langle v, \partial_{s} u_{\rho}\right\rangle d s \text { and } \lim _{k \rightarrow \infty} \int_{0}^{t}\left\langle u, \partial_{s} v_{\rho}^{k}\right\rangle d s=\int_{0}^{t}\left\langle u, \partial_{s} v_{\rho}\right\rangle d s .
$$


Noting that the function $J$ defined in Lemma 3.6 is an even function, we have

$$
\begin{aligned}
& \int_{0}^{t}\left\langle v, \partial_{s} u_{\rho}\right\rangle d s+\int_{0}^{t}\left\langle u, \partial_{s} v_{\rho}\right\rangle d s \\
= & \int_{0}^{t} \int_{0}^{t} \partial_{s} J_{\rho}(s-\tau)\langle u(\tau), v(s)\rangle d s d \tau+\int_{0}^{t}\left\langle u, \partial_{s} v_{\rho}\right\rangle d s \\
= & -\int_{0}^{t} \int_{0}^{t} \partial_{\tau} J_{\rho}(s-\tau)\langle u(\tau), v(s)\rangle d s d \tau+\int_{0}^{t}\left\langle u, \partial_{s} v_{\rho}\right\rangle d s \\
= & -\int_{0}^{t} \int_{0}^{t} \partial_{\tau} J_{\rho}(\tau-s)\langle u(\tau), v(s)\rangle d s d \tau+\int_{0}^{t}\left\langle u, \partial_{s} v_{\rho}\right\rangle d s, \\
= & -\int_{0}^{t}\left\langle\partial_{\tau} v_{\rho}, u\right\rangle d \tau+\int_{0}^{t}\left\langle u, \partial_{s} v_{\rho}\right\rangle d s=0 .
\end{aligned}
$$

With the help of Lemma 3.6 and (3.12), we obtain

$$
\begin{gathered}
\lim _{\rho \rightarrow 0} \lim _{k \rightarrow \infty} \int_{0}^{t}\left\langle\Lambda^{\alpha} v, \Lambda^{\alpha} u_{\rho}^{k}\right\rangle d s=\int_{0}^{t}\left\langle\Lambda^{\alpha} v, \Lambda^{\alpha} u\right\rangle d s, \\
\lim _{k \rightarrow \infty}\left\langle v(t), u_{\rho}^{k}(t)\right\rangle d s=\left\langle v(t), u_{\rho}(t)\right\rangle,
\end{gathered}
$$

where the notation $\lim _{\rho \rightarrow 0} \lim _{k \rightarrow \infty}$ means that one first passes to the limit of $k$ and then that of $\rho$.

Thanks to the $L^{2}$ weak continuity of weak solutions proved in Theorem E.1 in appendix A, we deduce

$$
\begin{aligned}
\left\langle v(t), u_{\rho}(t)\right\rangle & =\left\langle v(t), \int_{0}^{t} \frac{1}{\rho} J\left(\frac{t-s}{\rho}\right) u(s) d s\right\rangle \\
& =\left\langle v(t), \int_{0}^{\frac{t}{\rho}} J\left(s^{\prime}\right) u\left(t-\rho s^{\prime}\right) d s^{\prime}\right\rangle \\
& =\left\langle v(t), \int_{0}^{1} J\left(s^{\prime}\right) u(t) d s\right\rangle+\int_{0}^{1} J\left(s^{\prime}\right)\left\langle v(t), u\left(t-\rho s^{\prime}\right)-u(t)\right\rangle d s^{\prime} \\
& =\frac{1}{2}\langle v(t), u(t)\rangle+o(\rho)
\end{aligned}
$$

where we have used the fact that $\int_{0}^{1} J_{\rho}(z) d z=\frac{1}{2}$ and $\rho<t$, which yields

$$
\lim _{\rho \rightarrow 0}\left\langle v(t), u_{\rho}(t)\right\rangle=\frac{1}{2}\langle v(t), u(t)\rangle .
$$

Likewise,

$$
\begin{aligned}
& \lim _{\rho \rightarrow 0} \lim _{k \rightarrow \infty} \int_{0}^{t}\left\langle\Lambda^{\alpha} u, \Lambda^{\alpha} v_{\rho}^{k}\right\rangle d s=\int_{0}^{t}\left\langle\Lambda^{\alpha} u, \Lambda^{\alpha} v\right\rangle d s, \\
& \lim _{\rho \rightarrow 0} \lim _{k \rightarrow \infty}\left\langle u(t), v_{\rho}^{k}(t)\right\rangle=\frac{1}{2}\langle v(t), u(t)\rangle, \\
& \lim _{\rho \rightarrow 0} \lim _{k \rightarrow \infty}\left\langle u(0), v_{\rho}^{k}(0)\right\rangle=\frac{1}{2}\langle u(0), v(0)\rangle, \\
& \lim _{\rho \rightarrow 0} \lim _{k \rightarrow \infty}\left\langle v(0), u_{\rho}^{k}(0)\right\rangle=\frac{1}{2}\langle v(0), u(0)\rangle .
\end{aligned}
$$


Consequently, taking the limit in (3.13) leads to

$$
\begin{aligned}
& \langle u(t), v(t)\rangle+2 \int_{0}^{t}\left\langle\Lambda^{\alpha} u, \Lambda^{\alpha} v\right\rangle d s \\
= & \langle u(0), v(0)\rangle-\lim _{\rho \rightarrow 0} \lim _{k \rightarrow \infty} \int_{0}^{t}\left[\left\langle u \cdot \nabla u, v_{\rho}^{k}\right\rangle d s+\left\langle v \cdot \nabla v, u_{\rho}^{k}\right\rangle\right] d s .
\end{aligned}
$$

It remains to pass to the limit in nonlinear terms in (3.14). In order to do this, using the Gagliardo-Nirenberg inequality

$$
\|\nabla u\|_{L^{\frac{3}{\alpha}}} \leq C\left\|\Lambda^{\alpha} u\right\|_{L^{2}}^{\frac{6 \alpha-5}{2 \alpha}}\left\|\Lambda^{2 \alpha} u\right\|_{L^{2}}^{\frac{5-4 \alpha}{2 \alpha}},
$$

and

$$
\left\|v_{\rho}^{k}-v\right\|_{L^{\frac{6}{3-2 \alpha}}}^{2} \leq C\left\|\Lambda^{\alpha}\left(v_{\rho}^{k}-v\right)\right\|_{L^{2}}^{2}
$$

we see that

$$
\begin{aligned}
& \int_{0}^{t}\left\langle u \cdot \nabla u, v_{\rho}^{k}-v\right\rangle d s \\
\leq & \left(\int_{0}^{t}\|u \cdot \nabla u\|_{L^{\frac{6}{3+2 \alpha}}}^{2} d s\right)^{\frac{1}{2}}\left(\int_{0}^{t}\left\|v_{\rho}-v\right\|_{L^{\frac{6}{3-2 \alpha}}}^{2} d s\right)^{\frac{1}{2}} \\
\leq & \left(\int_{0}^{t}\|u\|_{L^{2}}^{2}\|\nabla u\|_{\frac{3}{\alpha}}^{2} d s\right)^{\frac{1}{2}}\left(\int_{0}^{t}\left\|v_{\rho}^{k}-v\right\|_{L^{\frac{6}{3-2 \alpha}}}^{2} d s\right)^{\frac{1}{2}} \\
\leq & \left(\int_{0}^{t}\|u\|_{L^{2}}^{2}\left\|\Lambda^{\alpha} u(t)\right\|_{L^{2}}^{\frac{6 \alpha-5}{\alpha}}\left\|\Lambda^{2 \alpha} u(t)\right\|_{L^{2}}^{\frac{5-4 \alpha}{\alpha}} d s\right)^{\frac{1}{2}}\left(\int_{0}^{t}\left\|\Lambda^{\alpha}\left(v_{\rho}^{k}-v\right)\right\|_{L^{2}}^{2} d s\right)^{\frac{1}{2}},
\end{aligned}
$$

where we have used the Hölder inequality.

Utilizing the Hölder inequality and the Gagliardo-Nirenberg inequality

$$
\|v\|_{L^{\frac{12}{4 \alpha+1}}} \leq C\|v\|_{L^{2}}^{\frac{8 \alpha-5}{4 \alpha}}\left\|\Lambda^{\alpha} v\right\|_{L^{\frac{5-4 \alpha}{4 \alpha}}}^{\frac{5+4}{2 \alpha}}
$$

and

$$
\left\|\nabla\left(u_{\rho}^{k}-u\right)\right\|_{L^{\frac{6}{5-4 \alpha}}} \leq C\left\|\Lambda^{2 \alpha}\left(u_{\rho}^{k}-u\right)\right\|_{L^{2}},
$$

by integration by parts, we infer that

$$
\begin{aligned}
& \int_{0}^{t}\left\langle v \cdot \nabla v, u_{\rho}^{k}-u\right\rangle d s \\
= & -\int_{0}^{t}\left\langle v \cdot \nabla\left(u_{\rho}^{k}-u\right), v\right\rangle d s \\
\leq & \int_{0}^{t}\left\|v^{2}\right\|_{L^{\frac{6}{4 \alpha+1}}}\left\|\nabla\left(u_{\rho}^{k}-u\right)\right\|_{L^{\frac{6}{5-4 \alpha}}} d s \\
\leq & \int_{0}^{t}\|v\|_{L^{\frac{12}{4 \alpha+1}}}^{2}\left\|\nabla\left(u_{\rho}^{k}-u\right)\right\|_{L^{\frac{6}{5-4 \alpha}}} d s \\
\leq & \left(\int_{0}^{t}\|v\|_{L^{2}}^{\frac{8 \alpha-5}{\alpha}}\left\|\Lambda^{\alpha} v\right\|_{L^{2}}^{\frac{5-4 \alpha}{\alpha}} d s\right)^{\frac{1}{2}}\left(\int_{0}^{t}\left\|\Lambda^{2 \alpha}\left(u_{\rho}^{k}-u\right)\right\|_{L^{2}}^{2} d s\right)^{\frac{1}{2}} .
\end{aligned}
$$


In the light of $\frac{5-4 \alpha}{\alpha} \leq 2$, we can pass the limit in (3.15) and (3.16), namely,

$$
\begin{aligned}
& \lim _{\rho \rightarrow 0} \lim _{k \rightarrow \infty} \int_{0}^{t}\left\langle u \cdot \nabla u, v_{\rho}^{k}\right\rangle d s=\int_{0}^{t}\langle u \cdot \nabla u, v\rangle d s, \\
& \lim _{\rho \rightarrow 0} \lim _{k \rightarrow \infty} \int_{0}^{t}\left\langle v \cdot \nabla v, u_{\rho}^{k}\right\rangle d s=-\int_{0}^{t}\langle v \cdot \nabla u, v\rangle d s .
\end{aligned}
$$

Consequently, we have

$$
\lim _{\rho \rightarrow 0} \lim _{k \rightarrow \infty} \int_{0}^{t}\left[\left\langle u \cdot \nabla u, v_{\rho}^{k}\right\rangle+\left\langle v \cdot \nabla v, u_{\rho}^{k}\right\rangle\right] d s=\int_{0}^{t}\langle w \cdot \nabla u, v\rangle d s .
$$

Using the integration by parts, we know that

$$
\int_{0}^{t}\left\langle w \cdot \nabla u, u_{\rho}^{k}\right\rangle d s=-\int_{0}^{t}\left\langle w \cdot \nabla u_{\rho}^{k}, u\right\rangle d s .
$$

Exactly as in the derivation of (3.17) and (3.18), we deduce that

$$
\lim _{\rho \rightarrow 0} \lim _{k \rightarrow \infty} \int_{0}^{t}\left\langle w \cdot \nabla u, u_{\rho}^{k}\right\rangle d s=-\int_{0}^{t}\langle w \cdot \nabla u, u\rangle d s,
$$

and

which implies

$$
\lim _{\rho \rightarrow 0} \lim _{k \rightarrow \infty} \int_{0}^{t}\left\langle w \cdot \nabla u_{\rho}^{k}, u\right\rangle d s=\int_{0}^{t}\langle w \cdot \nabla u, u\rangle d s,
$$

$$
\int_{0}^{t}\langle w \cdot \nabla u, u\rangle d s=0 .
$$

Combining (3.14) and (3.19) with (3.20), we obtain (3.11).

Proof of Proposition 3.4. In view of (3.11) and the energy inequality corresponding to $s=0$ in (E.2), straightforward calculations show that

$$
\begin{aligned}
& \|w(t)\|_{L^{2}}^{2}+2 \int_{0}^{t}\left\|\Lambda^{\alpha} w(s)\right\|_{L^{2}}^{2} d s \\
= & \|u(t)\|_{L^{2}}^{2}+\|v(t)\|_{L^{2}}^{2}-2\langle u(t), v(t)\rangle+2 \int_{0}^{t}\left\|\Lambda^{\alpha} u(s)\right\|_{L^{2}}^{2} d s \\
& +2 \int_{0}^{t}\left\|\Lambda^{\alpha} v(s)\right\|_{L^{2}}^{2} d s-4 \int_{0}^{t}\left\langle\Lambda^{\alpha} u(s), \Lambda^{\alpha} v(s)\right\rangle d s \\
\leq & \|u(0)\|_{L^{2}}^{2}+\|v(0)\|_{L^{2}}^{2}-2\langle u(0), v(0)\rangle-2 \int_{0}^{t}\langle w(s) \cdot \nabla w(s), u(s)\rangle d s \\
= & -2 \int_{0}^{t}\langle w(s) \cdot \nabla u(s), w(s)\rangle d s .
\end{aligned}
$$

By the Hölder inequality, the Gagliardo-Nirenberg inequality used in (3.16) and the Young's inequality, we get

$$
\begin{aligned}
-\int_{0}^{t}\langle w(s) \cdot \nabla u(s), w(s)\rangle d s & \leq \int_{0}^{t}\left\|w^{2}(s)\right\|_{L^{\frac{6}{4 \alpha+1}}}\|\nabla u(s)\|_{L^{\frac{6}{5-4 \alpha}}} d s \\
& \leq \int_{0}^{t}\|w(s)\|_{L^{2}}^{\frac{8 \alpha-5}{2 \alpha}}\left\|\Lambda^{\alpha} w(s)\right\|_{L^{2}}^{\frac{5-4 \alpha}{2 \alpha}}\left\|\Lambda^{2 \alpha} u(s)\right\|_{L^{2}} d s \\
& \leq \int_{0}^{t}\left\|\Lambda^{\alpha} w(s)\right\|_{L^{2}}^{2} d s+C \int_{0}^{t}\|w(s)\|_{L^{2}}^{2}\left\|\Lambda^{2 \alpha} u(s)\right\|_{L^{2}}^{\frac{4 \alpha}{8 \alpha-5}} d s .
\end{aligned}
$$


This together with (3.21) yields

$$
\|w(t)\|_{L^{2}}^{2}+\int_{0}^{t}\left\|\Lambda^{\alpha} w(s)\right\|_{L^{2}}^{2} d s \leq C \int_{0}^{t}\|w(s)\|_{L^{2}}^{2}\left\|\Lambda^{2 \alpha} u(s)\right\|_{L^{2}}^{\frac{4 \alpha}{8 \alpha-5}} d s .
$$

Thus, making use of Gronwall's Lemma, we accomplish the proof.

\section{Eventual regularity and Hausdorff dimension estimate}

We shall basically follow the pathway of $[\mathbf{8}$, Section 6$]$ to complete the proof of Theorem 2.4 and 2.6 in this section.

4.1. Eventual regularity. This subsection focuses on the eventual regularity of weak solutions, which means that there exists a $T^{*}>0$ such that every weak solution $u(t)$ is a strong solution on $\left(T^{*}, \infty\right)$.

Proof of TheOrem 2.6. By the global well-posedness result for small solution in Proposition 3.1 and Theorem 6.1 in [27], it suffices to prove that there exists a $T^{*}>0$ (maybe large) such that

$$
\left\|u\left(T^{*}\right)\right\|_{L^{2}}^{\frac{6 \alpha-5}{2 \alpha}}\left\|\Lambda^{\alpha} u\left(T^{*}\right)\right\|_{L^{2}}^{\frac{5-4 \alpha}{2 \alpha}}<C_{1}^{-1} \nu .
$$

The proof of $(4.1)$ is easy. Otherwise, for any $t \in(0, \infty)$, we have

$$
\|u(t)\|_{L^{2}}^{\frac{6 \alpha-5}{2 \alpha}}\left\|\Lambda^{\alpha} u(t)\right\|_{L^{2}}^{\frac{5-4 \alpha}{2 \alpha}} \geq C_{1}^{-1} \nu .
$$

By means of energy inequality (the strong energy inequality (2.1) for $\tau=0$ ) $\left(\|u(0)\|_{L^{2}} \geq\|u(t)\|_{L^{2}}\right)$, we obtain the uniform low bound of $\left\|\Lambda^{\alpha} u(t)\right\|_{L^{2}}$. But this contradicts with the energy inequality (the strong energy inequality (2.1) for $\tau=0)$.

From the above discussion, we could construct a strong solutions $\tilde{u}$ with the initial data $u\left(T^{*}\right)$. Together with strong energy inequality $(2.1)$

$$
\|u(t)\|_{L^{2}}^{2}+2 \nu \int_{T^{*}}^{t}\left\|\Lambda^{\alpha} u(s)\right\|_{L^{2}}^{2} d s \leq\left\|u\left(T^{*}\right)\right\|_{L^{2}}^{2}, t \geq T^{*},
$$

and Proposition 3.4, we get $\tilde{u}(t)=u(t)$ a.e. on $\left(T^{*}, \infty\right)$. This concludes the proof of Theorem 2.6.

4.2. Hausdorff dimension estimate. Based on the eventual regularity of weak solutions, we know that the possible singular time points of weak solutions to (1.5) must be contained in finite interval $\left(0, T^{*}\right)$. With the help of Proposition 3.1 and Proposition 3.4, we have

LEMma 4.1. The regular point set $\mathcal{R}$ can be decomposed as follows

$$
\mathcal{R}=\left(\bigcup_{i \in A}\left(\tau_{i}, s_{i}\right)\right) \bigcup\left(T^{*}, \infty\right), s_{i} \in \mathcal{I} \mathcal{R}
$$

where $\left(\tau_{i}, s_{i}\right) \bigcap\left(\tau_{j}, s_{j}\right)=\emptyset$ for $i \neq j$ and the set $A$ is at most countable, where $T^{*}$ is same as in (4.1). Furthermore, the Lebesgue measure of the irregular points set $\mathcal{I R}$ of the weak solution on time is zero.

Proof. Note that the weak solution $u$ belongs to $L^{2}\left(\left(0, T^{*}\right) ; H^{\alpha}\right)$ and satisfies strong energy inequality (2.1)

$$
\|u(t)\|_{L^{2}}^{2}+2 \nu \int_{\tau}^{t}\left\|\Lambda^{\alpha} u(s)\right\|_{L^{2}}^{2} d s \leq\|u(\tau)\|_{L^{2}}^{2}, \tau=0 \text { or a.e. } \tau \geq 0 \text { and } t \in\left[0, T^{*}\right) .
$$


Therefore, for a.e. $\tau \in\left(0, T^{*}\right)$, the weak solution $u(t)$ is a strong one on some interval $(\tau, \tau+T(\tau))$ with the initial data $u(\tau)$ due to Proposition 3.1 and Proposition 3.4. The interval $(\tau, \tau+T(\tau))$ can be extended to a maximal one $\left(\tau^{\prime}, s^{\prime}\right)$ containing $(\tau, \tau+T(\tau))$ such that $s^{\prime} \in \mathcal{I} \mathcal{R}$. Thence, $\mathcal{R}=\left(\bigcup_{i \in A}\left(\tau_{i}, s_{i}\right)\right) \cup\left(T^{*}, \infty\right)$, where $\left(\tau_{i}, s_{i}\right) \bigcap\left(\tau_{j}, s_{j}\right)=\emptyset$ for $i \neq j$ and the set $\mathrm{A}$ is at most countable since the set composed by mutually disjoint open intervals belonging to the line is finite or countable. Claim (4.2) is proved.

Denote $I=\left\{s \in\left(0, T^{*}\right) \mid u(s) \in H^{\alpha}\right\}$. It is clear that

$$
\left|\left(0, T^{*}\right) \backslash I\right|=\left|I \backslash\left(\bigcup_{i \in A}\left(\tau_{i}, s_{i}\right)\right)\right|=0 .
$$

The Lebesgue measure of the $\mathcal{I} \mathcal{R}$ is zero.

It should be point out that the proof of the endpoint case $\alpha=5 / 6$ in Theorem 2.4 has been achieved based on the fact that the 1 dimensional Hausdorff measure coincides with Lebesgue measure on $\mathbb{R}$. Consequently, the following proof focuses its attention on the regime $5 / 6<\alpha<5 / 4$. In order to conclude the rest part of proof of Theorem 2.4, we need exploit the necessary condition for $t_{0}$ to be a possible irregular point to generalized Navier-Stokes equations (1.5) similar to the Navier-Stokes equations

$$
\|\nabla u(t)\|_{L^{2}} \geq \frac{\nu^{3 / 4} C}{\left(t_{0}-t\right)^{1 / 4}}, t<t_{0}
$$

appearing in Leray's groundbreaking paper $[\mathbf{1 1}]$.

As mentioned in Remark 3.3, the key inequality (3.4) help us to obtain

Proposition 4.2. Assume that $t_{0}$ is an irregular point of a weak solution $u$. Then

$$
\lim _{t \rightarrow t_{0}}\left\|\Lambda^{\alpha} u(t)\right\|_{L^{2}}=\infty
$$

Furthermore, for $5 / 6<\alpha<5 / 4$, there holds

$$
\left\|\Lambda^{\alpha} u(t)\right\|_{L^{2}} \geq \frac{\nu^{\frac{5-2 \alpha}{4 \alpha}} C}{\left(t_{0}-t\right)^{\frac{6 \alpha-5}{4 \alpha}}}, t<t_{0} .
$$

Proof. If (4.3) was not true, we can pick up $t_{k}$ such that $t_{k} \rightarrow t_{0}$ as $k \rightarrow \infty$ with $t_{k}<t_{0}$, moreover,

$$
\left\|\Lambda^{\alpha} u\left(t_{k}\right)\right\|_{L^{2}} \leq C_{2} .
$$

Thanks to Proposition 3.1, Theorem 3.4 and the strong energy inequality, proceeding as before, the weak solution $u(t)$ can be seen as a strong solution with the initial data $u\left(t_{k}\right)$ on $\left(t_{k}, t_{k}+T\left(t_{k}\right)\right)$, where

$$
T\left(t_{k}\right)=\frac{\nu^{\frac{5-2 \alpha}{6 \alpha-5}}}{\frac{2 \alpha C}{6 \alpha-5}\left\|\Lambda^{\alpha} u\left(t_{k}\right)\right\|_{L^{2}}^{\frac{4 \alpha}{6 \alpha-5}}} \geq C_{2}^{-\frac{4 \alpha}{6 \alpha-5}} C_{3}=T_{0},
$$

where $T_{0}$ does not depend on $k$.

We can choose $t_{k^{\prime}}$ such that $t_{k^{\prime}}+T_{0}>t_{0}$, so the weak solution $u(t)$ is a strong solution on $\left(t_{k}, t_{k}+T_{0}\right)$, which is a contradiction to the fact that $t_{0}$ is an irregular point of the weak solution. Thus (4.3) holds true. 

obtain

Integrating the inequality (3.4) with respect with time variable on $[t, \tau)$, we

$$
\frac{1}{\left\|\Lambda^{\alpha} u(t)\right\|_{L^{2}}^{\frac{4 \alpha}{6 \alpha-5}}}-\frac{1}{\left\|\Lambda^{\alpha} u(\tau)\right\|_{L^{2}}^{\frac{4 \alpha}{6 \alpha-5}}} \leq C \nu^{\frac{2 \alpha-5}{6 \alpha-5}}(\tau-t), t<\tau<t_{0} .
$$

Let $\tau \rightarrow t_{0}$ and (4.3) yields that

$$
\left\|\Lambda^{\alpha} u(t)\right\|_{L^{2}} \geq \frac{\nu^{\frac{5-2 \alpha}{4 \alpha}} C}{\left(t_{0}-t\right)^{\frac{6 \alpha-5}{4 \alpha}}}
$$

which proves (4.4).

Finally, we are ready to prove Theorem 2.4.

Proof of TheOrem 2.4. It follows from the energy inequality (the strong energy inequality (2.1) for $\tau=0$ ) that

$$
\sum_{i \in A} \int_{\tau_{i}}^{s_{i}}\left\|\Lambda^{\alpha} u(s)\right\|_{L^{2}}^{2} d s \leq C\|u(0)\|_{L^{2}}^{2}
$$

where $A$ is defined as in Lemma 4.1 .

With the help of (4.4), we infer that

$$
\sum_{i \in A} \int_{\tau_{i}}^{s_{i}}\left\|\Lambda^{\alpha} u(s)\right\|_{L^{2}}^{2} d s \geq C \sum_{i \in A} \int_{\tau_{i}}^{s_{i}} \frac{1}{\left(s_{i}-s\right)^{\frac{6 \alpha-5}{2 \alpha}}} d s \geq C \sum_{i \in A}\left(s_{i}-\tau_{i}\right)^{\frac{5-4 \alpha}{2 \alpha}} .
$$

Therefore, for any $\varepsilon>0$, there exists a finite part $A_{1}$ of $A$ such that

$$
\sum_{i \in A \backslash A_{1}}\left(s_{i}-\tau_{i}\right)^{\frac{5-4 \alpha}{2 \alpha}}<\varepsilon
$$

We denote the finite interval $\left(0, T^{*}\right) \backslash\left(\bigcup_{i \in A_{1}}\left(\tau_{i}, s_{i}\right)\right)=\bigcup_{j=1}^{N} r_{j}$.

We note that

$$
\bigcup_{j=1}^{N} r_{j}=\mathcal{I R} \bigcup\left(\bigcup_{i \in A \backslash A_{1}}\left(\tau_{i}, s_{i}\right)\right)
$$

Using $|\mathcal{I} \mathcal{R}|=0$, we find

$$
\operatorname{diam}\left\{r_{j}\right\}=\sum_{\substack{i \in A \backslash A_{1} \\\left(\tau_{i}, s_{i}\right) \subset r_{j}}}\left(s_{i}-\tau_{i}\right) \leq \sum_{i \in A \backslash A_{1}}\left(s_{i}-\tau_{i}\right) .
$$

Direct estimates give

$$
\begin{aligned}
\sum_{j=1}^{N} \operatorname{diam}\left\{r_{j}\right\}^{\frac{5-4 \alpha}{2 \alpha}} & =\sum_{j=1}^{N}\left(\sum_{\substack{i \in A \backslash A_{1} \\
\left(\tau_{i}, s_{i}\right) \subset r_{j}}}\left(s_{i}-\tau_{i}\right)\right)^{\frac{5-4 \alpha}{2 \alpha}} \\
& \leq \sum_{j=1}^{N}\left(\sum_{\substack{i \in A \backslash A_{1} \\
\left(\tau_{i}, s_{i}\right) \subset r_{j}}}\left(s_{i}-\tau_{i}\right)^{\frac{5-4 \alpha}{2 \alpha}}\right) \\
& =\sum_{i \in A \backslash A_{1}}\left(s_{i}-\tau_{i}\right)^{\frac{5-4 \alpha}{2 \alpha}}<\varepsilon .
\end{aligned}
$$

In view of Definition 2.3, the proof is complete. 


\section{Appendix A. Existence of weak solutions}

To make our paper more self-contained and more readable, we outline the existence proof of weak solutions to (1.5). The existence of weak solutions of global weak solutions to the generalized MHD equations has been established by $\mathrm{Wu}$ in [25]. Contrary to Wu's work, we will modify some critical estimate. In addition, we show that the weak solutions satisfy the strong energy inequality and $L^{2}$ weak continuity.

TheOREM E.1. Let $u(0)$ be a divergence-free vector fields with finite energy. For any $T>0$, there exists a weak solution to (1.5) with $\alpha>0$ in the following sense

(1) $u \in L^{\infty}\left((0, T) ; L^{2}\right) \cap L^{2}\left((0, T) ; H^{\alpha}\right)$.

(2) $u$ solves (1.5) in the sense of distributions, namely,

$$
\int_{0}^{T}\left\langle u, \phi_{t}\right\rangle-\nu\left\langle\Lambda^{\alpha} u, \Lambda^{\alpha} \phi\right\rangle-\langle u \cdot \nabla u, \phi\rangle d t=-\langle u(0), \phi(0)\rangle
$$

for any $\phi \in C_{0}^{\infty}((0, T) \times \Omega)$.

(3) $u$ is $L^{2}$ weakly continuous on the interval $[0, T)$, that is,

$$
\lim _{t \rightarrow t_{0}}\left\langle u(t)-u\left(t_{0}\right), \varphi\right\rangle=0, \text { for all } t \in(0, T) \text { and all } \varphi \in L^{2}(\Omega)
$$

and $L^{2}$ strongly continuous at time 0 , namely,

$$
\lim _{t \rightarrow 0}\|u(t)-u(0)\|_{L^{2}}=0 .
$$

(4) $u$ verifies the strong energy inequality

$$
\|u(t)\|_{L^{2}}^{2}+2 \nu \int_{\tau}^{t}\left\|\Lambda^{\alpha} u(s)\right\|_{L^{2}}^{2} d s u \leq\|u(\tau)\|_{L^{2}}^{2},
$$

$\tau=0$ or a.e. $\tau>0$, and each $t \in[\tau, T)$.

Proof. We will apply the Galerkin method in the periodic domain similar to the classical Friedrich's method in the whole space to construct an approximate solution sequence. Let us define the operator $P_{N}$ by

$$
P_{N} f(x)=\mathcal{F}^{-1}\left(1_{k \leq N}(k) \hat{u}(k)\right)=\sum_{|k| \leq N} e^{i k \cdot x} \hat{u}(k)=\sum_{|k| \leq N} \int_{\Omega} e^{i k \cdot\left(x-x^{\prime}\right)} u\left(x^{\prime}\right) d x^{\prime} .
$$

We seek approximate solution $u^{N}=\sum_{|k| \leq N} e^{i k \cdot x} \hat{u}(k)$ satisfy the following equations

$$
\partial_{t} u^{N}+\nu \Lambda^{2 \alpha} u^{N}+P_{N}\left(u^{N} \cdot \nabla u^{N}\right)+P_{N} \nabla p^{N}=0, \operatorname{div} u^{N}=0,
$$

together with the initial condition

$$
u^{N}(x, 0)=P_{N} u(x, 0) .
$$

This system can be viewed as an ordinary differential equations on $L^{2}$. The CauchyLipschitz theorem for ordinary differential system gives us the existence of a positive maximal time $T_{n}$ such that this system has a unique solution $u^{N} \in C\left(\left[0, T_{n}\right] ; L^{2}\right)$. According to the finite time blow-up theorem for ordinary differential system, it suffices to show the uniform energy estimates on $u^{N}$, which yields $T_{n}=T$ for an arbitrary but fixed $T>0$. 
Multiplying (E.3) by $u^{N}$ and integrating by parts, we see that

$$
\frac{1}{2} \frac{d}{d t}\left\|u^{N}(t)\right\|_{L^{2}}^{2}+\left\|\Lambda^{\alpha} u^{N}(t)\right\|_{L^{2}}^{2}=0, t \leq T,
$$

which implies that

$$
\frac{1}{2}\left\|u^{N}(t)\right\|_{L^{2}}^{2}+\int_{0}^{t}\left\|\Lambda^{\alpha} u^{N}(s)\right\|_{L^{2}}^{2} d s=\frac{1}{2}\left\|u^{N}(0)\right\|_{L^{2}}^{2} \leq \frac{1}{2}\|u(0)\|_{L^{2}}^{2},
$$

This yields that the $L^{2}$ norm of $u^{N}$ is controlled and $T_{n}=T$. From the last inequality, by a diagonalization process, we can find a subsequence, again denoted by $u^{N}$, such that $u^{N} \stackrel{*}{\rightarrow} u$ in $L^{\infty}\left((0, T) ; L^{2}\right)$ and $u^{N} \rightarrow u$ in $L^{2}\left((0, T) ; L^{2}\right)$ as $N \rightarrow \infty$.

Just as the Navier-Stokes equations, one key point is to obtain a strong convergence in $L^{2}\left((0, T) ; L^{2}\right)$ to pass to the limit in the nonlinear term. The Aubin-Lions ([22, Theorem 2.1, p.184]) Lemma allows us to achieve this. Thence, we turn to bound $\partial_{t} u^{N}$ via the equation

$$
\partial_{t} u^{N}=\nu \Lambda^{2 \alpha} u^{N}-P_{N}\left(u^{N} \cdot \nabla u^{N}\right)-P_{N} \nabla p^{N} .
$$

By Hölder's inequality, integrating by parts and (E.4), for any $h \in L^{2}\left((0, T) ; H^{3}\right)$, we find that

$$
\begin{aligned}
\int_{0}^{T}\left\langle\Lambda^{2 \alpha} u^{N}(s), h(s)\right\rangle d s & \leq \int_{0}^{T}\left\|\Lambda^{\alpha} u^{N}(s)\right\|_{L^{2}}\left\|\Lambda^{\alpha} h(s)\right\|_{L^{2}} d s \\
& \leq\left(\int_{0}^{T}\left\|\Lambda^{\alpha} u^{N}(s)\right\|_{L^{2}}^{2} d s\right)^{1 / 2}\left(\int_{0}^{T}\left\|\Lambda^{\alpha} h(s)\right\|_{L^{2}}^{2} d s\right)^{1 / 2} \\
& \leq\|u(0)\|_{L^{2}}\|h\|_{L^{2}\left((0, T) ; H^{3}\right)} .
\end{aligned}
$$

Applying Hölder's inequality and (E.4), we see that

$$
\begin{aligned}
& \int_{0}^{T}\left\langle P_{N}\left(u^{N}(s) \cdot \nabla u^{N}(s)\right), h(s)\right\rangle d s \\
\leq & \int_{0}^{T}\left\|u^{N}(s)\right\|_{L^{2}}\left\|u^{N}(s)\right\|_{L^{\frac{6}{3-2 \alpha}}}\|\nabla h(s)\|_{L^{\frac{3}{\alpha}}} d s \\
\leq & C\left\|u^{N}\right\|_{L^{\infty}\left((0, T) ; L^{2}\right)}\left\|\Lambda^{\alpha} u^{N}\right\|_{L^{2}\left((0, T) ; L^{2}\right)}\|h\|_{L^{2}\left((0, T) ; H^{3}\right)} \\
\leq & C\|u(0)\|_{L^{2}}\|h\|_{L^{2}\left((0, T) ; H^{3}\right)},
\end{aligned}
$$

where we used the Sobolev embedding $H^{\alpha} \hookrightarrow L^{\frac{6}{3-2 \alpha}}$ and $H^{2} \hookrightarrow L^{\frac{3}{\alpha}}$.

To bound $P_{N} \nabla p^{N}$, we take divergence of (E.3) to obtain the pressure equation

$$
\Delta P_{N} p^{N}=-\sum_{i, j} \partial_{i} \partial_{j} P_{N}\left(u_{i}^{N} u_{j}^{N}\right)
$$

In the light of the classical Calderón-Zygmund Theorem, repeating the deduction process of (E.6), we deduce that

$$
\begin{aligned}
\int_{0}^{T}\left\langle P_{N} \nabla p^{N}(s), h(s)\right\rangle d s & \leq \int_{0}^{T}\left\|p^{N}(s)\right\|_{L^{\frac{3}{3-\alpha}}}\|\nabla h(s)\|_{L^{\frac{3}{\alpha}}} d s \\
& \leq C \int_{0}^{T} \mid u^{N}(s)\left\|_{L^{2}}\right\| u^{N}(s)\left\|_{L^{\frac{6}{3-2 \alpha}}}\right\| \nabla h(s) \|_{L^{\frac{3}{\alpha}}} d s \\
& \leq C\|u(0)\|_{L^{2}}\|h\|_{L^{2}\left((0, T) ; H^{3}\right)} .
\end{aligned}
$$


Combining these estimates, we obtain

$$
\partial_{t} u^{N} \in L^{2}\left((0, T) ; H^{-3}\right)
$$

which together with (E.4) yields that

$$
u^{N} \rightarrow u \text { in } L^{2}\left((0, T) ; L^{2}\right) .
$$

Taking the scalar product of (E.3) with $\phi \in C_{0}^{\infty}((0, T) \times \Omega)$ and integrating, we have

$$
\int_{0}^{T}\left\langle u^{N}, \phi_{s}\right\rangle-\nu\left\langle\Lambda^{\alpha} u^{N}, \Lambda^{\alpha} \phi\right\rangle-\left\langle u^{N} \cdot \nabla u^{N}, \phi\right\rangle d s=-\left\langle u^{N}(0), \phi(0)\right\rangle .
$$

Now we can pass to the limit in the above equations exactly as the standard NaveriStokes equations (for example [22, p.196]) to show (E.1).

In order to prove property (3), we shall apply Arzelà-Ascoli theorem to pass to the limit

$$
\lim _{N \rightarrow \infty}\left\langle u^{N}(t), \varphi\right\rangle=\langle u(t), \varphi\rangle, \text { for each } t \in[0, T], \varphi \in L^{2}(\Omega),
$$

hence, it is enough to show that the function

$$
f_{N}(t)=\left\langle u^{N}(t), \varphi\right\rangle
$$

is uniformly bounded and equicontinuous on $[0, T]$.

It follows from (E.4) that $f_{N}(t)$ is uniformly bounded. Notice that $\varphi$ is independent of time, arguing in the same manner as (E.8),

$$
\begin{aligned}
\left|f_{N}\left(t_{1}\right)-f_{N}\left(t_{2}\right)\right| & \leq\left|\int_{t_{2}}^{t_{1}}\left\langle\partial_{s} u^{N}(s), \varphi\right\rangle d s\right| \\
& \leq C\left|t_{1}-t_{2}\right|^{1 / 2}\left\|\partial_{s} u^{N}\right\|_{L^{2}\left((0, T) ; H^{-3}\right)}\|\varphi\|_{H^{3}} \\
& \leq C\left|t_{1}-t_{2}\right|^{1 / 2}\|u(0)\|_{L^{2}}\|\varphi\|_{H^{3}},
\end{aligned}
$$

which implies that $f_{N}(t)$ equicontinuous when $\varphi \in H^{3}$. In the light of Arzelà-Ascoli theorem, we infer that

$$
\lim _{N \rightarrow \infty}\left\langle u^{N}(t), \varphi\right\rangle=\langle u(t), \varphi\rangle, \text { for each } t \in[0, T],
$$

and $f(t)=\langle u(t), \varphi\rangle$ is continuous function where $\varphi \in H^{3}$. With the help of the fact that $H^{3}$ is dense in $L^{2}$, by dense argument, we conclude that the weak solutions is $L^{2}$ weakly continuous.

Making use of (E.9), we see that

(E.10) $\|u(t)\|_{L^{2}}=\sup _{\|v\|_{L^{2}} \leq 1}\langle u(t), v\rangle=\sup _{\|v\|_{L^{2}} \leq 1} \liminf _{N \rightarrow \infty}\langle u(t), v\rangle \leq \liminf _{N \rightarrow \infty}\left\|u^{N}(t)\right\|_{L^{2}}$,

for any $t \in[0, T]$, which together with (E.4) implies

$$
\lim _{t \rightarrow 0}\|u(t)\|_{L^{2}} \leq\|u(0)\|_{L^{2}} .
$$

Similar to (E.10), utilize the $L^{2}$ weak continuity to obtain

$$
\|u(0)\|_{L^{2}}=\sup _{\|v\|_{L^{2}} \leq 1}\langle u(0), v\rangle=\sup _{\|v\|_{L^{2}} \leq 1} \liminf _{t \rightarrow 0}\langle u(t), v\rangle \leq \liminf _{t \rightarrow 0}\|u(t)\|_{L^{2}} .
$$

Recalling that parallelogram identity holds in Hilbert space $L^{2}$, we know that $\lim _{t \rightarrow 0}\|u(t)\|_{L^{2}}=\|u(0)\|_{L^{2}}$ and $L^{2}$ weak continuity mean that $\lim _{t \rightarrow 0}\|u(t)-u(0)\|_{L^{2}}=0$. 
At last, we check that the energy inequality corresponding to $s=0$ in (E.2) and the strong energy inequality (E.2) are valid. On the one hand, it follows from (E.4), (E.10) and Fatou's Lemma that

$$
\|u(t)\|_{L^{2}}^{2}+2 \nu \int_{0}^{t}\left\|\Lambda^{\alpha} u(s)\right\|_{L^{2}}^{2} d s \leq\|u(0)\|_{L^{2}}^{2}, t \in(0, T) .
$$

On the other hand, there exists a set $E \subset[0, T)$ whose Lebesgue measure is zero and a subsequence (still denoted by the same symbol) $u^{N}$ such that

$$
u^{N}(\tau) \rightarrow u(\tau) \text { as } N \rightarrow \infty \text { in } L^{2} \text {, for each } \tau \in[0, T) / E
$$

due to the fact that $u^{N}(t) \in L^{2}\left([0, T) ; H^{\alpha}\right)$ and $H^{\alpha} \hookrightarrow \hookrightarrow L^{2}$. Based on this, we can pass to the limit of

$$
\frac{1}{2}\left\|u^{N}(t)\right\|_{L^{2}}^{2}+\nu \int_{\tau}^{t}\left\|\Lambda^{\alpha} u^{N}(s)\right\|_{L^{2}}^{2} d s=\frac{1}{2}\left\|u^{N}(\tau)\right\|_{L^{2}}^{2},
$$

to arrive at

$$
\frac{1}{2}\|u(t)\|_{L^{2}}^{2}+\nu \int_{\tau}^{t}\left\|\Lambda^{\alpha} u(s)\right\|_{L^{2}}^{2} d s \leq \frac{1}{2}\|u(\tau)\|_{L^{2}}^{2},
$$

for almost every $\tau \in[0, T)$ and each $t \in[\tau, T)$.

Acknowledgements: The authors would like to express their sincere gratitude to Gang Wu and Jiefeng Zhao for many helpful discussions on this topic.

\section{References}

1. Á. Bényi and T. Oh, The Sobolev inequality on the torus revisited, Publ. Math. Debrecen. 83 (2013), 359-374

2. L. Caffarelli, R. Kohn and L. Nirenberg, Partial regularity of suitable weak solutions of NavierStokes equation, Comm. Pure. Appl. Math. 35 (1982), 771-831

3. M. Cannone, C. Miao and G. Wu, On the inviscid limit of the two-dimensional Navier-Stokes equations with fractional diffusion, Adv. Math. Sci. Appl. 18 (2008), 607-624

4. Q. Chen, C. Miao and Z. Zhang, On the uniqueness of weak solutions for the $3 D$ NavierStokes equations, Ann. Inst. H. Poincaré Anal. Non Linéaire. 26 (2009), 2165-2180

5. M. Dabkowski, Eventual regularity of the solutions to the supercritical dissipative quasigeostrophic equation, Geom. Funct. Anal. 21 ( 2011), 1-13

6. S. Dubois, Uniqueness for some Leray-Hopf solutions to the Navier-Stokes equations, J. Differential Equations. 189 (2003), 99-147

7. L. Escauriaza, G. A. Seregin and V. Šverák, On $L^{\infty} L^{3}$-solutions to the Navier-Stokes equations and Backward uniqueness, Russian Mathematical Surveys, 58 (2003), 211-250

8. G. P. Galdi, An Introduction to the Navier-Stokes Initial-Boundary Value Problem, Fundamental directions in mathematical fluid mechanics, Birkhäuser, Basel, (2000), 1-70

9. J. G. Heywood, The Navier-Stokes equations: on the existence, regularity and decay of solutions, Indiana Univ. Math. J. 29 (1980), 639-681

10. N. H. Katz and N. Pavlović, A cheap Caffarelli-Kohn-Nirenberg inequality for the NavierStokes equation with hyper-dissipation, Geom. Funct. Anal. 12 (2002), 355-379

11. J. Leray, Sur le mouvement déun liquide visqueux emplissant léspace, Acta Math. 63 (1934), $193-248$

12. J. L. Lions, Quelques résultats déxistence dans deséquations aux dérivés partielles non linéaires, Bull. Soc. Math. France, 87 (1959), 245-273

13. J. L. Lions, Quelques méthodes de resolution des problémes aux limites non linéaires, Vol 1. Dunod, Paris, 1969

14. K. Masuda, Weak solutions of Navier-Stokes equations, Tohoku Math. J. 36 (1984), 623-646

15. C. Miao and L. Xue, On the regularity of a class of generalized quasi-geostrophic equations, J. Differential Equations, 251 (2011), 2789-2821 
16. C. Miao, B. Yuan and B. Zhang, Well-posedness of the Cauchy problem for the fractional power dissipative equations, Nonlinear Anal. 68 (2008), 461-484

17. V. Scheffer, Turbulence and Hausdorff dimension, in Turbulence and the Navier-Stokes Equations, Lecture Notes in Math., Springer-Verlag, 565 (1976), 94-112

18. V. Scheffer, The Navier-Stokes equations on a bounded domain, Commun. Math. Phys., 73 (1980), 1-42

19. J. Serrin, The Initial Value Problem for the Navier-Stokes Equations, Nonlinear Problems, R.E. Langer Ed., Madison: University of Wisconsin Press. 1963

20. L. Silvestre, Eventual regularization for the slightly supercritical quasi-geostrophic equation, Ann. Inst. H. Poincaré Anal. Non Linéaire. 27 (2010), 693-704

21. R. Témam, Navier-Stokes Equations and Nonlinear Functional Analysis, 2nd Edition, SIAM, Philadelphia, 1995

22. R. Témam, Navier-Stokes equations: Theory and numerical analysis, AMS Chelsea Publishing, Providence, RI, 2001. Reprint of the 1984 edition.

23. T. Tao, Global regularity for a logarithmically supercritical hyperdissipative Navier-Stokes equation, Analysis \& PDE 3 (2009), 361-366

24. Y. Wang and $\mathrm{G}$. Wu, A unified proof on the partial regularity for suitable weak solutions of non-stationary and stationary Navier-Stokes equations, J. Differential Equations, 256 (2014), 1224-1249

25. J. Wu, Generalized MHD equations, J. Differential Equations, 195 (2003), 284-312

26. J. Wu, The generalized incompressible Navier-Stokes equations in Besov spaces, Dynamics of PDE, 1 (2004), 381-400

27. J. Wu, Lower bounds for an integral involving fractional Laplacians and the generalized Navier-Stokes equations in Besov spaces, Commun. Math. Phys., 263 (2006), 803-831

28. J. Wu, Global regularity for a class of generalized magnetohydrodynamic equations, Journal of Mathematical Fluid Mechanics, 13 (2011), 295-305

29. W. P. Ziemer, Weakly differentiable functions. Sobolev spaces and Functions of Bounded Variation, Graduate texts in math. 120. Springer-Verlag. New York. 1989

School of Mathematical Sciences, Capital Normal University, Beijing 100048, P. R. CHINA

E-mail address: jiuqs@mail.cnu.edu.cn

School of Mathematical Sciences, Capital Normal University, Beijing 100048, P. R. CHINA

E-mail address: wangyanqing20056@gmail.com 